Unsteady flow in a rotating torus after a sudden change in rotation rate

Hewitt, R.E. and Hazel, A.L. and Clarke, R.J. and Denier, J.P.

2011

MIMS EPrint: 2013.60

Manchester Institute for Mathematical Sciences

School of Mathematics

The University of Manchester

\footnotetext{
Reports available from: http://eprints.maths.manchester.ac.uk/

And by contacting: The MIMS Secretary

School of Mathematics

The University of Manchester

Manchester, M13 9PL, UK
} 


\title{
Unsteady flow in a rotating torus after a sudden change in rotation rate
}

\author{
R. E. Hewitt ${ }^{1} \dagger$, A. L. Hazel ${ }^{1}$, R. J. Clarke ${ }^{2}$ and J. P. Denier ${ }^{3}$ \\ ${ }^{1}$ School of Mathematics, The University of Manchester, Oxford Road, Manchester M13 9PL, UK \\ 2 Department of Engineering Science, University of Auckland, New Zealand \\ ${ }^{3}$ School of Mathematical Sciences, University of Adelaide, Adelaide 5005, Australia
}

(Received 19 December 2010; revised 16 May 2011; accepted 30 August 2011; first published online 20 October 2011)

\begin{abstract}
We consider the temporal evolution of a viscous incompressible fluid in a torus of finite curvature; a problem first investigated by Madden \& Mullin (J. Fluid Mech., vol. 265,1994 , pp. 265-217). The system is initially in a state of rigid-body rotation (about the axis of rotational symmetry) and the container's rotation rate is then changed impulsively. We describe the transient flow that is induced at small values of the Ekman number, over a time scale that is comparable to one complete rotation of the container. We show that (rotationally symmetric) eruptive singularities (of the boundary layer) occur at the inner or outer bend of the pipe for a decrease or an increase in rotation rate respectively. Moreover, on allowing for a change in direction of rotation, there is a (negative) ratio of initial-to-final rotation frequencies for which eruptive singularities can occur at both the inner and outer bend simultaneously. We also demonstrate that the flow is susceptible to a combination of axisymmetric centrifugal and non-axisymmetric inflectional instabilities. The inflectional instability arises as a consequence of the developing eruption and is shown to be in qualitative agreement with the experimental observations of Madden \& Mullin (1994). Throughout our work, detailed quantitative comparisons are made between asymptotic predictions and finite- (but small-) Ekman-number Navier-Stokes computations using a finite-element method. We find that the boundary-layer results correctly capture the (finite-Ekman-number) rotationally symmetric flow and its global stability to linearised perturbations.
\end{abstract}

Key words: boundary-layer stability, pipe flow boundary layer

\section{Introduction}

The content of this paper is directly motivated by the work of Madden \& Mullin (1994) (hereinafter referred to as MM), which considered the internal flow induced by the sudden rotation of a toroidal container filled with an incompressible Newtonian fluid. Their work was largely experimental in nature, capturing the flow response by a combination of flow visualisation and laser-Doppler velocimetry methods. Numerical results were also presented, but computational restrictions were such that they could not be directly related to the experimental data across the full parameter range studied.

†Email address for correspondence: richard.e.hewitt@manchester.ac.uk 
Subsequent to a rapid linear ramp of the frequency of the container, from a state of rest to a specified angular frequency, a number of features were noted by MM. At sufficiently large values of the Reynolds number (based on the mean speed of the torus and its (minor) radius), the flow rapidly became disordered, but did so through a welldefined sequence of events. In the initial stages an axisymmetric 'front' was observed to develop at the outermost radius of the toroidal pipe. This front propagated radially inwards away from the wall, before small-scale waves appeared in the around-torus direction, breaking the rotational symmetry of the flow. The rapid growth of these waves led ultimately to three-dimensional disordered flow (figure 19 of their paper presents a sequence of flow visualisation pictures that nicely captures the evolution, as obtained by a light sheet through the mid-plane of symmetry of the container).

The discussion in MM suggests that, for sufficiently rapid rotation, a second instability is present at the inner boundary. They argue that a centrifugal instability is to be expected on the basis of Rayleigh's criterion, but of course some care is needed because the criterion only applies for steady, inviscid, axisymmetric swirl flows. MM speculate that the origin and break-up of the inwardly propagating front has two potential sources (pp. 241-242): a collisional boundary-layer phenomenon and a centrifugal instability; however no detailed comparisons were possible to support either source. In particular, regarding the non-axisymmetric waves, MM propose that a growing centrifugal instability developing at the inner wall could be advected in the secondary meridional flow, moving in the cross-sectional plane to the outer wall where it eventually leads to the wave-like instability.

Rather than restricting attention to the 'spin-up from rest' case considered by $\mathrm{MM}$, we address a broader range of problems in which a transition is made from a rigid-body rotation at an initial frequency, $\Omega_{i}$, to a second frequency, $\Omega_{f}$, such that $\left|\Omega_{i} / \Omega_{f}-1\right|=O(1)$. (Throughout this work we choose $\Omega_{f}$ as the natural frequency; in cases of spin-down to rest $\left(\Omega_{f}=0\right)$ one would choose $\Omega_{i}$ as the natural frequency.) The corresponding 'spin-down to rest' problem $\left(\Omega_{f}=0\right)$ was later described experimentally by del Pino et al. (2008). Again, propagating fronts were observed (albeit this time at the inner bend of the torus) and a purely local analysis showed that a singular eruption of the boundary layer can be qualitatively linked to the experimental data. In this work we will extend the problems of both MM and del Pino et al. (2008) to general values of the ratio $\Omega_{i} / \Omega_{f}$, describe the full boundary-layer solution around the entirety of the container wall, and assess the linear stability of the induced flow to both axisymmetric and non-axisymmetric perturbations. Our aim is to clarify the origin of the fronts and waves observed by MM by an analysis that combines asymptotic (boundary-layer) methods with Navier-Stokes computations.

In curved pipes, any axial flow down the length of the pipe must be associated with a corresponding cross-sectional flow in the meridional plane through an interplay of viscous drag, centrifugal forcing and pressure gradients. At large Reynolds numbers (small Ekman numbers), this coupling leads to a boundary-layer behaviour that is often referred to as 'collisional', with meridional flows around the inside of the pipe meeting on the reflectional plane of symmetry. In the steady entry-flow problem in a curved pipe, Stewartson, Cebeci \& Chang (1980) showed that, in the large-Dean-number limit, a collisional structure must develop at the inner bend of the pipe at a finite distance downstream from the entry. This collisional structure is associated with a finite-distance singularity in the wall boundary layer at the inner equatorial point of the pipe. Equally one can consider the unsteady fully developed analogue of this problem, as discussed by Cowley, Van Dommelen \& Lam (1990). Again a singular boundary-layer response is achieved at the inner bend of the pipe, but in this case at 
a finite time. The finite-time singularity of the unsteady flow has many similarities to the corresponding unsteady singular response described by Banks \& Zaturska (1979) and Simpson \& Stewartson (1982) for an impulsively rotated sphere. The singularity in these problems is interpreted as being directly associated with an eruption of the boundary layer into the interior core flow.

The configuration that we discuss herein is a special case of more general curvedpipe flows, a topic that has received a great deal of attention owing mostly to its relevance to a number of biological and industrial applications. In large part, these existing investigations have concentrated on either steady flow or pulsatile flow driven by an internal pressure gradient. Typically the geometry that has received the most attention is that of small curvature (a loosely coiled pipe) and the steady solution structure in the limits of small and large Dean number has been clarified, see the review by Berger, Talbot \& Yao (1983). Multiple solution branches are known to exist, see Yang \& Keller (1986), and recent work has clarified the effects of finite pipe curvature on the steady solution branches (Siggers \& Waters 2005) and also identified multiple periodic solutions (Siggers \& Waters 2008).

In contrast to the pressure-driven flows described above, the steady (initial) flow in a closed torus driven by constant boundary rotation is a rigid-body rotation, which greatly simplifies any analysis. Indeed, the flow configuration of MM provides a unique opportunity in which the evolution of a system is complicated by both eruptive boundary-layer singularities and instabilities, but nonetheless remains open to investigation by asymptotic methods, as well as providing a simple geometry for experimental and numerical investigation. The closed (periodic) nature of the flow domain, together with the initially axisymmetric response, and unambiguously defined boundary conditions make this problem a rare example in which boundarylayer singularities, hydrodynamic instabilities, nonlinear Navier-Stokes computations and experimental data can be combined without any requirement for ad-hoc modelling.

The format of this paper is as follows. In $\S 2$ (and the Appendix) we describe the coordinate system, governing parameters and boundary conditions for a system that begins in a rigid-body rotation, prior to an impulsive change in the rotation rate of the torus at $t=0$. In $\S 3$, we use boundary-layer theory to examine the axisymmetric flow response in the limit of asymptotically low Ekman number, over a time scale comparable to the rotation period of the container. The axisymmetric evolution of the entire flow field is considered in $\S 4$, by solving (numerically) the rotationally symmetric Navier-Stokes equations at finite (but small) Ekman numbers; and good agreement is found with the predictions made in $\S 3$. In $\S 5$, we consider the nonaxisymmetric (inviscid) instability mechanism for the transient boundary-layer flow using asymptotic methods combined with numerical Navier-Stokes solutions. Section 6 investigates the relevance of axisymmetric centrifugal instabilities, again via a combination of asymptotic methods and numerical simulation. Finally, our conclusions are given in $\S 7$.

\section{Formulation}

We consider a torus with a centreline (major) radius $L$ and a cross-sectional (minor) radius $a$, filled with an incompressible viscous fluid of kinematic viscosity $v$, see figure 1. The fluid and the container are initially in a state of rigid-body rotation at an angular frequency $\Omega_{i}$. At time $t=0$, an unsteady flow is generated by an impulsive change in the rotation rate of the torus to an angular frequency $\Omega_{f}$, where $\left|\Omega_{i} / \Omega_{f}-1\right|=O(1)$. The ultimate state of the system is a rigid-body rotation at the 


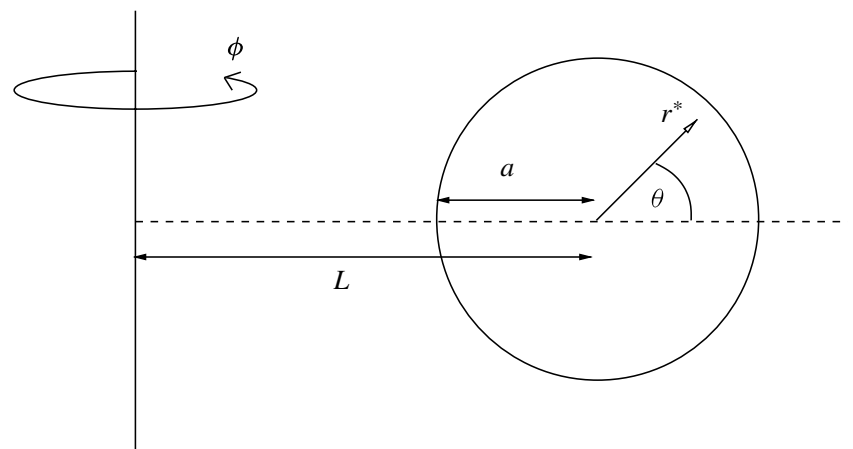

FIGURE 1. A cross-section of the torus, with dimensional centreline (major) radius $L$ and pipe radius $a$. The outermost point of the torus is at $\theta=0, r^{*}=a$, whilst the innermost point is at $\theta=\pi, r^{*}=a$. We use a dimensionless coordinate system in which $r=r^{*} / a$, which means that the dimensionless centreline (major) radius of the torus becomes $\delta^{-1}$, where $\delta=a / L$ is a curvature parameter.

new frequency $\Omega_{f}$, but our interest lies in the response of the fluid within the first few rotations of the container after $t=0$.

The flow response will depend on three parameters: (i) the curvature of the pipe $\delta=a / L$; (ii) the Ekman number (an inverse Reynolds number) $E k=v /\left(a^{2} \Omega_{f}\right)$; and (iii) the relative rotation ratio $\Omega_{r}=\Omega_{i} / \Omega_{f}$. We note that values of $\Omega_{r}>1$ correspond to spin-down flows, whereas $0<\Omega_{r}<1$ are spin-up flows. If $\Omega_{r}<0$ the direction of rotation reverses; a case sometimes referred to as 'spin over'.

It is most convenient to work in a toroidal coordinate system $(\operatorname{ar}, \theta, \phi)$ (see figure 1) and the appropriate dimensionless form of the Navier-Stokes equations is given in the Appendix, in which we have chosen $a$ as the typical length scale, $\Omega_{f} a$ as the typical velocity scale, $\Omega_{f}^{-1}$ as the time scale and the pressure is non-dimensionalised on the inertial scale. The dimensionless velocity components corresponding to the coordinates $(r, \theta, \phi)$ are labelled $(u, v, w)$ and the initial state of the system is rigid-body rotation:

$$
u \equiv v \equiv 0, \quad w=\Omega_{r} h(r, \theta) / \delta \quad \text { at } t=0,
$$

where $h(r, \theta)=1+\delta r \cos \theta$ is the (dimensionless) distance from the axis of rotation relative to the centreline radius $L$. The boundary conditions for $t>0$ are that the torus rotates at the new frequency:

$$
u=v=0, \quad w=h(1, \theta) / \delta \quad \text { on } r=1 .
$$

The experimental configuration of MM used a toroidal pipe of radius $a=16 \mathrm{~mm}$ and centreline radius of curvature of $L=125 \mathrm{~mm}$, which leads to a curvature parameter, $\delta=a / L=0.128$. We shall use this value in all curvature-dependent computations presented, but we have found that the results are not qualitatively sensitive to the choice of $\delta$, provided that it is $O(1)$.

We begin our investigation by considering the initial response to the change in rotation rate; namely, the development of an impulsively generated axisymmetric boundary layer, adjacent to the container wall, which forms the subject of the next section.

\section{Rotationally symmetric $E k \ll 1$ boundary-layer evolution for $\boldsymbol{t}=\boldsymbol{O}(\mathbf{1})$}

In this section we address the boundary-layer problem by a combination of asymptotic and numerical methods. If the Ekman number is small then we anticipate 
that viscous effects should be initially confined to a thin boundary layer of width $E k^{1 / 2}$ adjacent to the torus wall. Indeed, for $t=O(1)$, the experimental results of MM indicate that the core fluid in the toroidal container can be assumed to be (initially) steady at leading order, being a rigid-body rotation at frequency $\Omega_{r}$. Consequently, the boundary-layer equations have a known pressure distribution in the core of the flow:

$$
p(r, \theta, t=0)=\Omega_{r}^{2} \cos \theta\left(r \delta^{-1}+\frac{1}{2} r^{2} \cos \theta\right),
$$

associated with the initial state of rigid-body rotation.

Throughout this section, we consider only rotationally symmetric motion, so all velocity components and the pressure are independent of $\phi$. The boundary layer is most concisely described by the velocity component along the pipe, $w(r, \theta, t)$, and a stream function $\psi(r, \theta, t)$ in the meridional plane:

$$
\begin{aligned}
u & =\frac{1}{h r} \frac{\partial(h \psi)}{\partial \theta}, \\
v & =-\frac{1}{h} \frac{\partial(h \psi)}{\partial r} .
\end{aligned}
$$

The leading-order, boundary-layer equations can now be obtained from (A $1 b)$ and (A 1c), as given in the Appendix, for a pressure that is constant across the layer and matches to the external distribution (3.1):

$$
\begin{aligned}
& w_{0 t}-\psi_{0 \theta} w_{0 \eta}+\psi_{0 \eta} w_{0 \theta}+\frac{1}{\varrho} \sin \theta \psi_{0} w_{0 \eta}-\frac{1}{\varrho} \sin \theta \psi_{0 \eta} w_{0}=w_{0 \eta \eta}, \\
& \psi_{0 \eta t}-\psi_{0 \theta} \psi_{0 \eta \eta}+\psi_{0 \eta} \psi_{0 \eta \theta}+\frac{1}{\varrho} \sin \theta \psi_{0} \psi_{0 \eta \eta}+\frac{1}{\varrho} \sin \theta w_{0}^{2}=\varrho \sin \theta \Omega_{r}^{2}+\psi_{0 \eta \eta \eta},
\end{aligned}
$$

where $\varrho(\theta)=\delta^{-1}+\cos (\theta)$ is the dimensionless distance from the axis of rotation. Here we have introduced the boundary-layer coordinate $\eta=(1-r) E k^{-1 / 2}=O(1)$, and the subscript zero indicates that these are the leading terms in a standard boundary-layer expansion

$$
\begin{gathered}
\psi=\psi_{0}(\eta, \theta, t) E k^{1 / 2}+\cdots, \\
w=w_{0}(\eta, \theta, t)+\cdots,
\end{gathered}
$$

where higher-order terms in $E k$ have been neglected. The initial conditions for the boundary layer are $w_{0}=\varrho \Omega_{r}, \psi_{0} \equiv 0$ at $t=0$, whilst the boundary conditions are $\psi_{0}=\psi_{0_{\eta}}=0, w_{0}=\varrho$ on $\eta=0$ and $w_{0} \rightarrow \varrho \Omega_{r}, \psi_{0_{\eta}} \rightarrow 0$ as $\eta \rightarrow \infty$.

Rather than solving (3.3) we prefer to transform the system to explicitly include the influence of pipe curvature and the structure of the secondary flow. We begin by making the following transformations:

$$
\begin{gathered}
\xi=\varrho(\theta)^{1 / 4} \eta, \\
\psi_{0}=\varrho(\theta)^{1 / 4} \Psi(\xi, \theta, t) \sin \theta, \\
w_{0}=\varrho(\theta) W(\xi, \theta, t) .
\end{gathered}
$$

It is convenient to include this low-curvature transformation, because then the steady solutions of the boundary-layer equations near $\theta=0, \pi$ are independent of the pipe curvature. The transformation also ensures that the centrifugal balance (which drives the secondary flow) is still achieved on setting $\delta=0$ in the resulting governing equations; although we will make no assumptions that $\delta$ is small in what follows. Our numerical solution of the boundary-layer equations will use parabolic marching, which 
requires an initial solution at the appropriate attachment point $\theta=0$ or $\pi$. Near these points (in a mid-plane symmetric flow) $\psi \sim \theta$ or $\pi-\theta$, and so it is convenient to introduce the $\sin \theta$ scaling above. (We use the terms attachment and detachment points by analogy with the stagnation points of attachment for flow over a bluff body, even though the flow in question here is a secondary flow in the meridional plane.)

Under the transformations (3.5), the equation (3.3) become

$$
\begin{aligned}
& \frac{1}{\sqrt{\varrho(\theta)}} W_{t}-W_{\xi}\left(\Psi \cos \theta+\Psi_{\theta} \sin \theta\right)+W_{\theta} \Psi_{\xi} \sin \theta \\
& +\frac{\sin ^{2} \theta}{4 \varrho(\theta)}\left(5 \Psi W_{\xi}-8 \Psi_{\xi} W\right)=W_{\xi \xi}, \\
& \frac{1}{\sqrt{\varrho(\theta)}} \Psi_{\xi t}+W^{2}-\Omega_{r}^{2}+\Psi_{\xi}\left(\Psi_{\xi} \cos \theta+\Psi_{\xi \theta} \sin \theta\right)-\Psi_{\xi \xi}\left(\Psi \cos \theta+\Psi_{\theta} \sin \theta\right) \\
& +\frac{\sin ^{2} \theta}{4 \varrho(\theta)}\left(5 \Psi_{\xi \xi} \Psi-2 \Psi_{\xi}^{2}\right)=\Psi_{\xi \xi \xi},
\end{aligned}
$$

subject to the boundary conditions

$$
\begin{aligned}
& W=1, \quad \Psi=\Psi_{\xi}=0, \quad \text { on } \xi=0, \\
& W \rightarrow \Omega_{r}, \quad \Psi_{\xi} \rightarrow 0, \quad \text { as } \xi \rightarrow \infty,
\end{aligned}
$$

for $t>0$, and initial conditions at $t=0$

$$
\Psi \equiv 0 \quad \text { and } \quad W \equiv \Omega_{r} .
$$

It is not possible to solve (3.6) subject to an impulsive change in rotation because there is a singular acceleration at $t=0$. The small-time structure is therefore explicitly built into the formulation by seeking a solution in terms of a Rayleigh-layer coordinate

$$
\begin{gathered}
y=t^{-1 / 2} \xi, \\
\Psi=t^{3 / 2} \bar{\Psi}(y, \theta, t), \\
W=\bar{W}(y, \theta, t),
\end{gathered}
$$

which ensures that at $t=0$ we have $O(1)$ solutions to $\bar{\Psi}$ and $\bar{W}$. In terms of these variables (3.6) become

$$
\begin{aligned}
& \frac{1}{\sqrt{\varrho(\theta)}}\left(t \bar{W}_{t}-\frac{y}{2} \bar{W}_{y}\right)-t^{2}\left[\bar{W}_{y}\left(\bar{\Psi} \cos \theta+\bar{\Psi}_{\theta} \sin \theta\right)-\bar{W}_{\theta} \bar{\Psi}_{y} \sin \theta\right. \\
& \left.-\frac{\sin ^{2} \theta}{4 \varrho(\theta)}\left(5 \bar{\Psi} \bar{W}_{y}-8 \bar{\Psi}_{y} \bar{W}\right)\right]=\bar{W}_{y y}, \\
& \frac{1}{\sqrt{\varrho(\theta)}}\left(t \bar{\Psi}_{y t}+\bar{\Psi}_{y}-\frac{y}{2} \bar{\Psi}_{y y}\right)+\bar{W}^{2}-\Omega_{r}^{2}+t^{2}\left[\bar{\Psi}_{y}\left(\bar{\Psi}_{y} \cos \theta+\bar{\Psi}_{y \theta} \sin \theta\right)\right. \\
& \left.-\bar{\Psi}_{y y}\left(\bar{\Psi} \cos \theta+\bar{\Psi}_{\theta} \sin \theta\right)+\frac{\sin ^{2} \theta}{4 \varrho(\theta)}\left(5 \bar{\Psi}_{y y} \bar{\Psi}-2 \bar{\Psi}_{y}^{2}\right)\right]=\bar{\Psi}_{y y y},
\end{aligned}
$$

with boundary and initial conditions given by the equivalent expressions to equations $(3.6 c)-(3.6 e)$.

It is now possible to solve the boundary-layer system for $t>0, \theta \in[0, \pi]$, $y \in[0, \infty)$ from an initial condition of rigid-body rotation, with an impulsive change in the container rotation rate applied at $t=0$. We solve (3.8) numerically for $t \in[0,1]$ 
then use the solution at $t=1$ as the initial data in the numerical solution of (3.6) for $t>1$. To begin the computation we require the initial data (for all $\theta \in[0, \pi]$ ), which are found by solving the leading-order form of (3.8); that is, we set $t=0$.

\subsection{The initial impulsive response for $t \ll 1$}

Some important features of this flow can be highlighted by examining the leadingorder response in the limit $t \ll 1$, which is determined from (3.8) by setting $t=0$. Under these conditions, the equations may be further rescaled to remove all explicit dependence on the curvature, which plays a lesser role in the developing Rayleigh layer because it is thin. The remaining terms in (3.8) will be retained under the rescaling $y=\varrho(\theta)^{1 / 4} \hat{Y}, \bar{\Psi}=\varrho(\theta)^{3 / 4} \hat{\Psi}$ and $\bar{W}=\hat{W}$, in which case

$$
\begin{gathered}
\psi=E k^{1 / 2} t^{3 / 2} \varrho(\theta) \hat{\Psi}(\hat{Y}) \sin \theta+\cdots, \\
w=\varrho(\theta) \hat{W}(\hat{Y})+\cdots,
\end{gathered}
$$

and $r=1-E k^{1 / 2} t^{1 / 2} \hat{Y}$. The leading-order equations are

$$
\begin{gathered}
-\frac{\hat{Y}}{2} \hat{W}_{\hat{Y}}=\hat{W}_{\hat{Y} \hat{Y}}, \\
\hat{\Psi}_{\hat{Y}}-\frac{\hat{Y}}{2} \hat{\Psi}_{\hat{Y} \hat{Y}}+\hat{W}^{2}-\Omega_{r}^{2}=\hat{\Psi}_{\hat{Y} \hat{Y} \hat{Y}},
\end{gathered}
$$

and the influence of the curvature of the toroidal container is entirely contained within the multiplying factors in the definitions (3.9). The boundary conditions are again given by expressions equivalent to (3.6c)-(3.6d).

We obtain the usual error-function solution for the axial flow:

$$
\hat{W}=1+\left(\Omega_{r}-1\right) \operatorname{Erf}\left(\frac{\hat{Y}}{2}\right),
$$

and although a corresponding solution for $\hat{\Psi}$ can be given, we do not do so here as it is not a compact expression. It is sufficient to note that we can determine $\hat{\Psi}_{\hat{Y}}(\hat{Y})$ and its contours are plotted as a function of the rotation ratio $\Omega_{r}$ and scaled boundary-layer coordinate $\hat{Y}$ in figure 2 . The quantity $\hat{\Psi}_{\hat{Y}}(\hat{Y})$ determines the meridional flow (in the $\theta$ direction) within the boundary layer for small times because

$$
v=t \varrho \sin \theta \hat{\Psi}_{\hat{Y}}+\cdots,
$$

for $t \ll 1$.

In figure 2 we show that for spin-down $\left(\Omega_{r}>1\right)$ the secondary flow in the boundary layer is uni-directional and directed from the outermost to the innermost radius of the container. Similarly for $\Omega_{r} \lesssim-0.56$ the flow remains uni-directional (again in the same outer-to-inner direction). For spin-up flows $0 \leqslant \Omega_{r} \leqslant 1$ the flow is unidirectional, but reversed so that the motion is from the innermost to the outermost radius of the container. However, there is a range of rotation ratio $-0.56 \lesssim \Omega_{r}<0$ where the initial response is more complicated, leading to bi-directional flow, with fluid going in both directions in the boundary layer. These different responses are induced by viscous acceleration/deceleration of near-wall fluid acting to break the centrifugal-pressure balance that exists in the initial rigid-body rotation. 


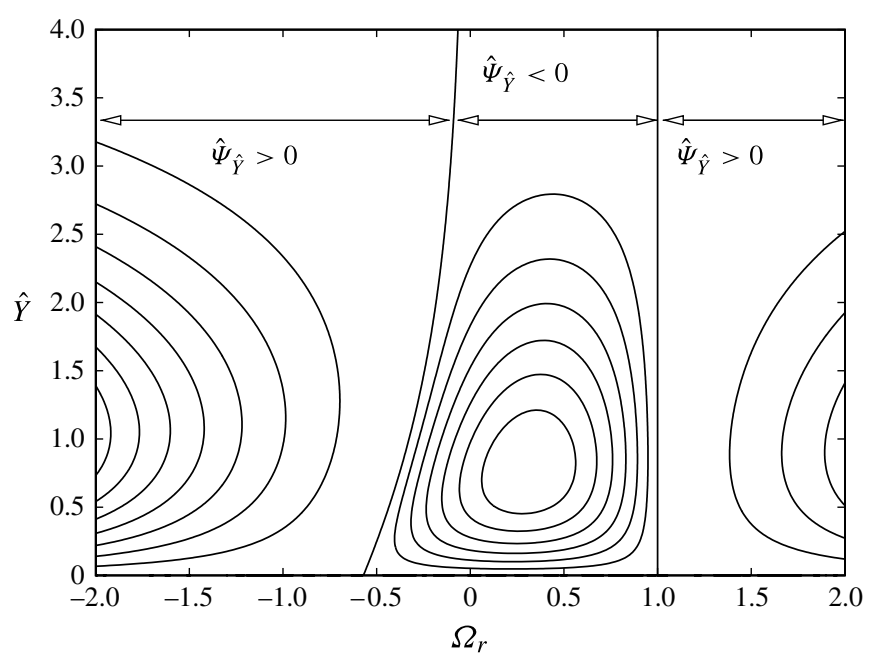

FIGURE 2. The azimuthal velocity in the meridional plane in the small-time limit. We show contours of $\hat{\Psi}_{\hat{Y}}$ as a function of $\Omega_{r}$. Contour levels are $1.6,1.4, \ldots, 0$ and $-0.02,-0.04, \ldots,-0.12 . \hat{\Psi}_{\hat{Y}}>0$ (for all $\hat{Y}$ ) corresponds to azimuthal flow in the boundary layer from the outer to inner radius of the container, and vice versa for $\hat{\Psi}_{\hat{Y}}<0$. The azimuthal flow changes sign at some $\hat{Y}>0$ for $-0.56 \lesssim \Omega_{r}<0$, meaning that the meridional flow is bi-directional in this region.

\subsection{Boundary-layer solutions at the attachment/detachment points, $\theta=0, \pi$}

As can be seen from the form of (3.6), at the attachment/detachment points of the meridional flow $\theta=0, \pi$, the terms $\Psi_{\theta}$ and $W_{\theta}$ vanish, allowing us to solve for the local flow independently of the behaviour at general values of $\theta$. In particular, it is possible to seek (quasi) steady solutions to the resulting equations:

$$
\begin{gathered}
-W_{\xi} \Psi \cos \theta=W_{\xi \xi}, \\
W^{2}-\Omega_{r}^{2}+\Psi_{\xi} \Psi_{\xi} \cos \theta-\Psi_{\xi \xi} \Psi \cos \theta=\Psi_{\xi \xi \xi},
\end{gathered}
$$

subject to

$$
\begin{aligned}
& W=1, \quad \Psi=\Psi_{\xi}=0, \quad \text { on } \xi=0, \\
& W \rightarrow \Omega_{r}, \quad \Psi_{\xi} \rightarrow 0, \quad \text { as } \xi \rightarrow \infty .
\end{aligned}
$$

For these equations to hold, we must have $\cos \theta= \pm 1$, corresponding to solutions local to the outermost $(\theta=0, r=1)$ and innermost $(\theta=\pi, r=1)$ positions of the torus respectively.

These solutions are quasi-steady in the sense that the effect of the boundary-layer transpiration on the (finite) interior core flow is neglected on the time scale of interest; that is, we assume that spin-up of the core fluid occurs over an asymptotically longer time scale (Benton \& Clark 1974).

The far-field asymptotic form of solutions to (3.12) is sufficient to show that steady solutions local to $\theta=0, \pi$ can only exist for $\Psi(\xi \rightarrow \infty) \cos \theta>0$, otherwise one finds exponential growth for large $\xi$. Therefore, we only expect quasi-steady solutions when the flow in the meridional plane is directed towards the boundary, that is, at an attachment point of the secondary flow. 


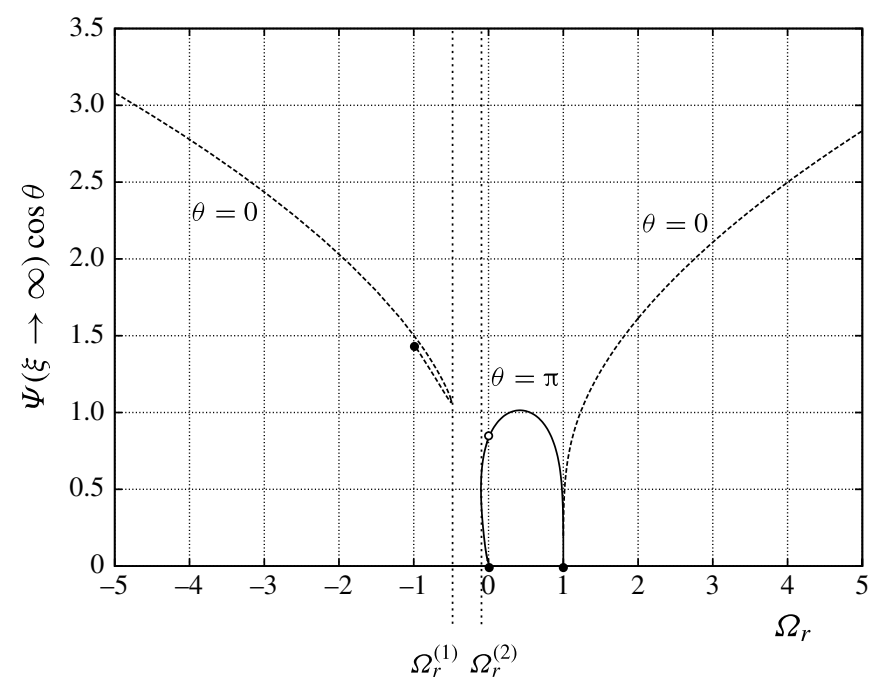

FIgURE 3. A measure of the radial mass flux into the boundary layer for the (quasi) steady equations (3.12) when $\theta=0, \pi$. The solid line represents solutions at $\theta=\pi$, the dashed lines are solutions at $\theta=0$. The filled data points correspond to the limiting values of $\Omega_{r}=1,0,-1$; a limiting structure of the boundary-layer system is found in each of these cases. The vertical lines denote the location of two limit points at $\Omega_{r}^{(1)} \approx-0.495$ and $\Omega_{r}^{(2)} \approx-0.101$. Spin-up from rest corresponds to $\Omega_{r}=0$, as indicated by the open circle, for which $\Psi(\xi \rightarrow \infty) \approx-0.833$. There are two additional solution branches with two associated limit points that exist in the region $-1.002<\Omega_{r}<-0.997$ (not visible on the scale of the figure). These results are independent of the choice of curvature $\delta$, which is a consequence of the transformation (3.5).

In figure 3 we characterise the (quasi) steady solutions by showing the far-field value of the stream function at the two locations $\theta=0, \pi$ obtained by solving the system (3.12) using a standard second-order finite-difference scheme. At these two positions in the flow $\Psi(\xi \rightarrow \infty)$ corresponds (with an appropriate $E k^{1 / 2}$ scaling) to the transpiration in the meridional plane induced by the boundary-layer solution.

We note that for spin-down $\Omega_{r}>1$ and spin-up $0 \leqslant \Omega_{r}<1$ there is a unique quasi-steady state. This quasi-steady behaviour is to be found at the outermost point of the torus for spin down and the innermost point for spin up.

For the cases of 'spin-over', i.e. $\Omega_{r}<0$, there are two solutions for $\Omega_{r}^{(2)}<\Omega_{r}<0$ and $-1 \lesssim \Omega_{r}<\Omega_{r}^{(1)}$ and a region of no (quasi) steady solutions for $\Omega_{r}^{(1)}<\Omega_{r}<\Omega_{r}^{(2)}$, where $\Omega_{r}^{(1)} \approx-0.495$ and $\Omega_{r}^{(2)} \approx-0.101$. We cannot completely rule out that steady solutions exist in the region $\Omega_{r}^{(1)}<\Omega_{r}<\Omega_{r}^{(2)}$ as no rigorous proof has been attempted, but a lengthy search has not revealed any. We find a very small region of parameter space near to $\Omega_{r} \approx-1$ for which four solutions can be achieved near the outermost wall of the torus. These additional states arise through a pair of limit points and occur over a parameter range that is too small to see in figure 3 .

A simple (linear perturbation) temporal eigenvalue analysis shows that all of the additional ('higher branch') states that arise from limit points (as shown in figure 3) are unstable to perturbations that have the same local boundary-layer structure. We do not believe that these additional solutions play an important role in the dynamics of the boundary layer for $t=O(1)$. 


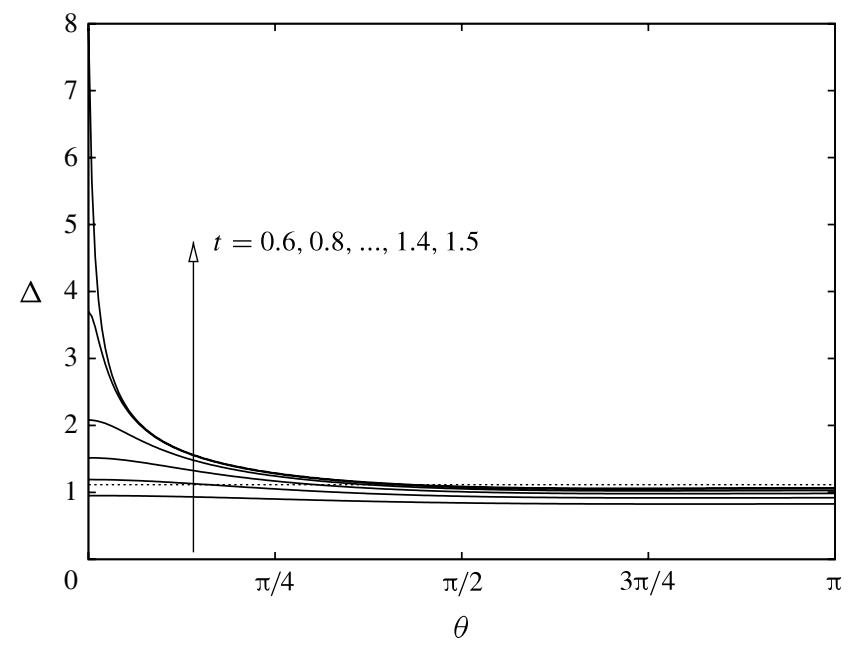

FIGURE 4. The evolution of the boundary-layer thickness $\Delta$, as defined in $(3.13 b)$, as a function of $\theta$ (around the inside of the toroidal container) for increasing time in the case of spin-up from rest $\left(\Omega_{r}=0\right)$. The boundary-layer solution suggests that a finite-time singularity develops in the neighbourhood of $\theta=0$. The dotted line shows the boundary-layer thickness of the quasi-steady steady local solution that can be developed in the region of $\theta=\pi$. The curvature is taken to be $\delta=0.128$.

\subsection{Numerical solution of the ( $\theta$-dependent) unsteady boundary layer}

We are now in a position to solve the $\theta$-dependent boundary-layer system (3.8) for $0 \leqslant t \leqslant 1$. The solution is obtained by (parabolically) marching in $\theta$ starting at the attachment point $(\theta=0$ or $\pi)$. The solution at this starting point is obtained numerically from the unsteady analogue of the solutions described in $\S 3.2$. The computation then proceeds on the three-dimensional mesh $\left\{\theta_{i}, y_{j}, t_{n}\right\}$ for the unknowns $\left\{\Psi_{i j}^{n}, W_{i j}^{n}\right\}$. We satisfy the equations at the central points $\frac{1}{2}\left(\theta_{i}+\theta_{i+1}\right), \frac{1}{2}\left(y_{j}+y_{j+1}\right)$, $\frac{1}{2}\left(t_{n}+t_{n+1}\right)$ and the resulting scheme is second order in both space and time. When the computation of (3.8) reaches $t=1$ it is convenient (and simple) to switch back to the unscaled system (3.6) for $t>1$.

As we have discussed in $\S 3.1$ and, in particular, figure 2 , if $\bar{\Psi}_{y}>0$ for all $y$ and $\theta$ we may (parabolically) march in the direction of increasing $\theta$ from $\theta=0$ to $\pi$; conversely, if $\bar{\Psi}_{y}<0$ we march from $\theta=\pi$ to $\theta=0$. If the boundary-layer flow in the meridional plane is bi-directional, then we cannot solve the system by this parabolic marching scheme, which precludes solution over the range $-0.56 \lesssim \Omega_{r}<0$, see $\S 3.1$.

The generic behaviour of the boundary-layer system (in cases for which the meridional flow is uni-directional) is highlighted in figures 4 and 5. The figures show a (scaled) measure of the boundary-layer thickness

$$
\begin{aligned}
\Delta(\theta, t) & =E k^{-1 / 2} \int_{r=0}^{r=1} \frac{\Omega_{r}-w(r, \theta, t) /\left(\delta^{-1}+r \cos \theta\right)}{\Omega_{r}-1} \mathrm{~d} r \\
& \approx \varrho(\theta)^{-1 / 4} \int_{0}^{\infty} \frac{\Omega_{r}-W(\xi, \theta, t)}{\Omega_{r}-1} \mathrm{~d} \xi,
\end{aligned}
$$

as a function of the angle $\theta$ as time evolves. The approximation in $(3.13 b)$ is to indicate that the leading-order boundary-layer solution is used. We list both definitions 


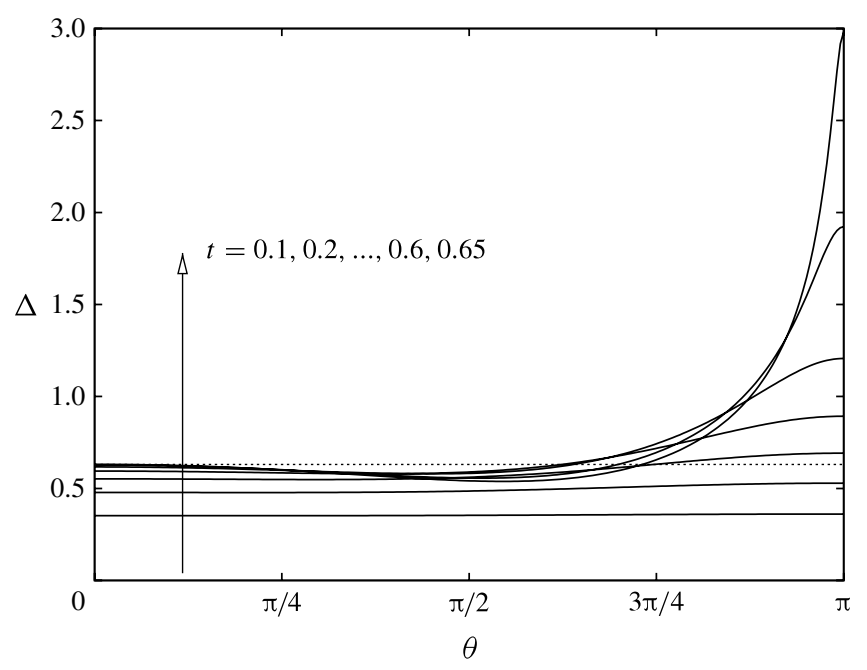

FIGURE 5. The evolution of the boundary-layer thickness $\Delta$, as defined in $(3.13 b)$, as a function of $\theta$ (around the inside of the toroidal container) for increasing time in the case of spin-down with $\Omega_{r}=2$. The boundary-layer solution suggests that a finite-time singularity is realised in the neighbourhood of $\theta=\pi$. The dotted line shows the boundary-layer thickness of the quasi-steady steady local solution that can be developed in the region of $\theta=0$. The curvature is taken to be $\delta=0.128$.

because we will later present detailed comparisons with finite-Ekman-number solutions of the Navier-Stokes equations, for which we use the definition (3.13a) instead. The factor $\delta^{-1}+r \cos \theta$ in (3.13a) is a dimensionless measure of the distance from the axis of rotation that is equivalent to the quantity $\varrho(\theta)$ when $r=1$.

In the case of spin-up from rest $\left(\Omega_{r}=0\right)$, we see in figure 4 that the response local to the innermost point of the torus wall $(\theta=\pi)$ approaches a quasi-steady state that is in agreement with the analysis of $\S 3.2$. However, at the outermost point of the torus wall $(\theta=0)$ the evolution is to what appears to be a singular response with an unbounded boundary-layer thickness obtained at a critical time (of approximately 1.55). In the case of spin-down (e.g. $\Omega_{r}=2$ ), as shown in figure 5 , we observe that the response is reversed, with a quasi-steady solution now being obtained at $\theta=0$ together with a singular response at $\theta=\pi$ (at approximately $t=0.7$ ).

\subsection{The finite-time singularity}

As shown in the previous section, there is strong evidence that the boundary-layer system evolves to a singularity local to the critical points $\theta=0$ and $\theta=\pi$. Which of these positions realises a singular response is dependent upon the rotation parameter $\Omega_{r}$. On setting $\sin \theta=0$ the singular response in system (3.6) can be connected to the asymptotic structure presented by Simpson \& Stewartson (1982). We do not reproduce the asymptotic description of the singular structure here and the interested reader is directed to their paper for the details; the structure is generic to such stagnation-point flows and does not have to be altered significantly to apply it to the problem under consideration here. As the asymptotic behaviour is known as $t \rightarrow t_{s}$ (where $t_{s}$ is the time of the singularity), we can estimate $t_{s}$ from the numerical solution of the governing system by fitting the data resulting from our computations of the unsteady boundary layer to the leading-order asymptotic form for $t_{s}-t \ll 1$. 


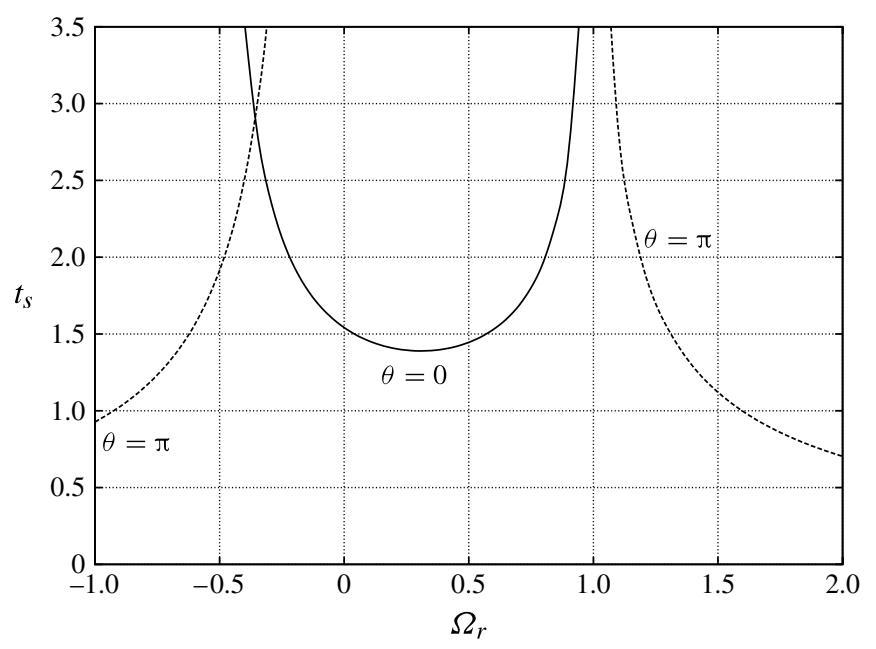

FIGURE 6. The behaviour of the breakdown time $t_{s}$ as a function of $\Omega_{r}$. The solid line represents a singularity at $\theta=0$, whilst the dashed line represents a singularity at $\theta=\pi$. We note that for $\Omega_{r} \approx-0.355$ a singularity can be found at both points $\theta=0$ and $\pi$ and occurs at approximately the same time. The curvature is taken to be $\delta=0.128$; however these results can be applied to other values of $\delta$ by a simple rescaling of $t_{s}$.

In figure 6 we show the functional dependence of the breakdown time $t_{s}$ on the rotation ratio $\Omega_{r}$ (for a curvature of $\delta=0.128$ ). In particular we notice that for spin-up from rest $\Omega_{r}=0$ the singularity occurs at $\theta=0$ at a time of approximately $t_{s} \approx 1.55$. For spin-down $\Omega_{r}=2$ the breakdown occurs at $\theta=\pi$ at a time of approximately $t_{s} \approx 0.7$.

Based on the evidence of numerical results, we conjecture that a singularity is found at $\theta=0, \pi$ whenever a steady solution does not exist for the chosen value of $\Omega_{r}$. Hence when $\Omega_{r}^{(1)} \approx-0.495<\Omega_{r}<\Omega_{r}^{(2)} \approx-0.101$, for which there is no steady solution at $\theta=0$ or $\theta=\pi$ (see figure 3 ), a singularity is obtained at both points. Furthermore, from figure 6 we see that at $\Omega_{r} \approx-0.355$ the time of breakdown is the same at both positions and the boundary-layer analysis predicts a simultaneous, double singularity.

\section{Rotationally symmetric Navier-Stokes computations}

We now consider direct numerical simulation of the Navier-Stokes equations at large, but finite, Ekman numbers in order to examine how the predictions arising from the boundary-layer theory, in particular the finite-time singularities, are realised in the full field equations.

Once again, we assume rotational symmetry, so that all three velocity components and the pressure are independent of $\phi$ and the equations are solved using an adaptive, Galerkin finite-element method implemented in the software library oomph-lib, see Heil \& Hazel (2006). The computational domain is the entire meridional cross-section of the torus. In other words, we do not assume any other additional symmetries in the system, but, in fact, all solutions remain symmetric about the mid-plane of the torus. The fluid variables are discretised using stable, isoparametric, $Q_{2} P_{1}$ (Taylor-Hood) elements in which the velocities are interpolated quadratically and the pressures are interpolated linearly within each element. The time derivative terms are treated 


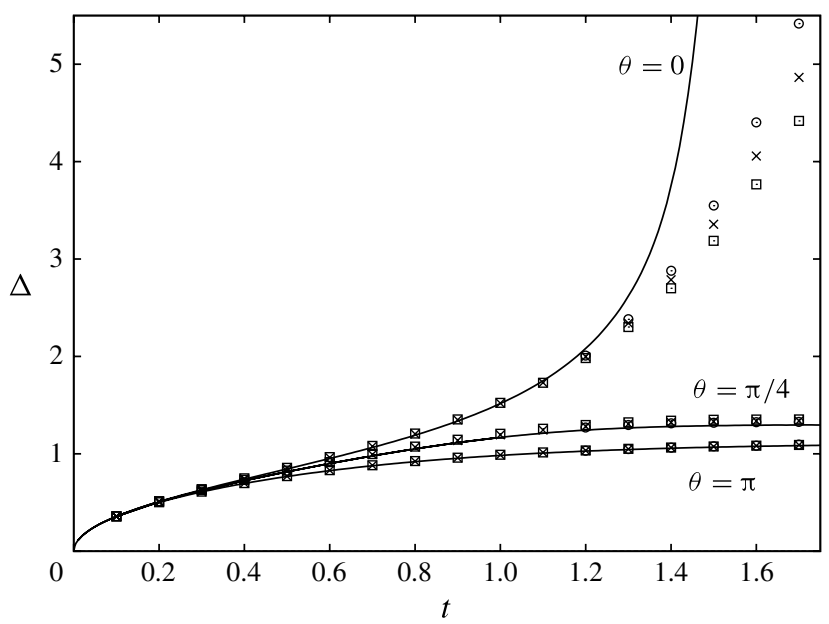

FIGURE 7. The evolution of the boundary-layer thickness $\Delta(\theta, t)$, as defined in (3.13), at the positions $\theta=0, \pi / 4, \pi$ for the case of spin-up from rest $\left(\Omega_{r}=0\right)$. The solid lines are solutions of the boundary-layer system whilst the data points are Navier-Stokes computations with $E k=1 / 1000$ (square), 1/2000 (cross) and 1/4000 (circle). The curvature is taken to be $\delta=0.128$.

implicitly using a second-order BDF2 method and the resulting discrete nonlinear system is solved by Newton-Raphson iteration; each linear system is solved iteratively by GMRES, using the LSC preconditioner described by Elman, Silvester \& Wathen (2005).

The approximate error in each element is calculated using an estimate based on the recovery of velocity gradients (Zienkiewicz \& Zhu 1992a,b). Elements in which the local estimated error is greater than $0.01 \%$ of the total estimated error across the entire domain are refined (into four new elements) and those for which it is less than $0.0001 \%$ are unrefined. This approach seeks to equipartition the error between the elements; and the total number of degrees of freedom can be adjusted by altering these tolerances or specifying a maximum level of refinement. Continuity of the solution is enforced by constraining the values at any hanging nodes, see Demkowicz et al. (1989). The typical time step used is $\mathrm{d} t=0.005$ (although a smaller value was used for convergence testing) and one spatial refinement was performed after each time step past the first. Typically the computation had approximately $3 \times 10^{5}$ degrees of freedom.

The Navier-Stokes computations begin at $t=\varepsilon$ with an initial condition given by the leading-order boundary-layer solution in the limit of small time, as presented in $\S 3.1$. Typically, we choose $\varepsilon=0.05$; and the results that we present herein have been verified to be independent of the choice of $\varepsilon \ll 1$. In both the Navier-Stokes and boundary-layer systems, varying the torus rotation rate gradually (rather than impulsively) does not alter the qualitative nature of the results, provided that the time scale of the transition in rotation rate is rapid relative to the time taken for the bulk of the fluid to spin up. Although in experiments the change in rotation rate must necessarily be gradual, we concentrate on the impulsive transition here because it avoids the introduction of any additional parameters into the problem.

We use the same measure of boundary-layer thickness $\Delta(\theta, t)$, defined in $(3.13 a)$, as a metric for the unsteady evolution of the flow; and present results for the time evolution of $\Delta$ at representative values of $\theta$ in the case of spin-up from rest $\left(\Omega_{r}=0\right)$ in figure 7 . We find excellent quantitative agreement between the 
boundary-layer predictions and the Navier-Stokes computations. In particular, the quasi-steady boundary-layer solution local to $\theta=\pi$ agrees well with the data for three different values of the Ekman number, $E k=1 / 1000,1 / 2000$ and 1/4000. More generally, the finite-Ekman-number data collapse well under the boundary-layer scaling implicit in $\Delta$, apart from near the breakdown event at $\theta=0$.

At $\theta=0$ (the outermost point of the torus), the sudden breakdown of the boundary layer is indeed realised at finite Ekman numbers, manifesting as a rapidly increasing boundary-layer thickness. The effect of the finite Ekman number is to delay the breakdown event somewhat because the radial pressure gradients that have been neglected in the boundary-layer system become reintroduced into the leading-order dynamics of the flow. Nonetheless, progressively decreasing the Ekman number leads to an increasingly rapid growth of the boundary-layer thickness corresponding to a dramatic eruption event.

Figure 8 plots the corresponding contours of axial frequency $w(r, \theta, t) /\left(\delta^{-1}+r \cos \theta\right)$ for $E k=1 / 2000$, showing the eruptive nature of the boundary layer at the outer wall of the pipe $(\theta=0)$. At $t=1.6$ the boundary layer at $\theta=0$ is approximately four times the thickness of that at $\theta=\pi$, and is rapidly increasing. By $t=2.2$ a significant quantity of more rapidly rotating fluid has been ejected into the static core near $\theta=0$ and the width of the region is no longer thin compared to the pipe radius.

To examine further the predictions of the boundary-layer theory we consider a case of 'spin-over', with $\Omega_{r}<0$. As highlighted in $\$ 3.4$, in this parameter regime we can find finite-time singularities at both $\theta=0$ and $\theta=\pi$, but the singularities do not, in general, occur at the same time. However, for the critical value $\Omega_{r}=-0.355$ (see figure 6) the boundary-layer singularities are predicted to occur simultaneously, leading to ejection into the core at both the inner and outer walls of the torus. In figure 9 we present the evolution of the boundary-layer thickness for this case, showing that, whilst the evolution at the intermediate point $\theta=\pi / 2$ is benign, the boundary-layer thickness at the equatorial points of the torus $\theta=0, \pi$ grows rapidly at the predicted time. Once again, as expected, the finite nature of the Ekman number eventually acts to mitigate the rate of growth of the boundary layer but does not inhibit it until the boundary layer is no longer thin compared to the pipe radius. No boundary-layer prediction is made at the $\theta=\pi / 2$ position owing to the bi-directional nature of the flow in the meridional plane making parabolic marching of (3.6) inappropriate. However, solutions local to the attachment/detachment points $\theta=0, \pi$ are still easily found from the unsteady analogue of (3.12) and are presented as the solid lines.

Figure 10 shows the same $\Omega_{r}=-0.355$ case, for $E k=1 / 2000$, as contours of constant axial frequency $w /\left(\delta^{-1}+r \cos \theta\right)$ at $t=3,4.5$. The simultaneous eruption at both $\theta=0$ and $\theta=\pi$ is visible, although the post-breakdown structure of the local flow is significantly different at each point; presumably a consequence of the different local structure of the collisional 'jets' and the interior pressure gradient.

Finally, in figure 11, we show the evolution of the axial frequency profile along the lines $\theta=0$, $\pi$ when $\Omega_{r}=-0.355$. Results from the boundary-layer and Navier-Stokes $(E k=1 / 1000)$ simulations corresponding to the same values of $\Delta$ (the measure of the boundary-layer thickness) are presented for varying $r$, where $r=1$ is the torus wall. Figure 11 $(a)$ shows the profile at the innermost $(\theta=\pi)$ point of the torus, whilst figure $11(b)$ shows the evolution at the outermost $(\theta=0)$ point. Although the effects of the finite Ekman number (obviously) mitigate the singular eruption, a crucial feature of the profiles that arises in the boundary-layer description remains in the Navier-Stokes results, namely the introduction of inflectional profiles in the axial flow. These inflection points in the neighbourhood of the breakdown event are a 

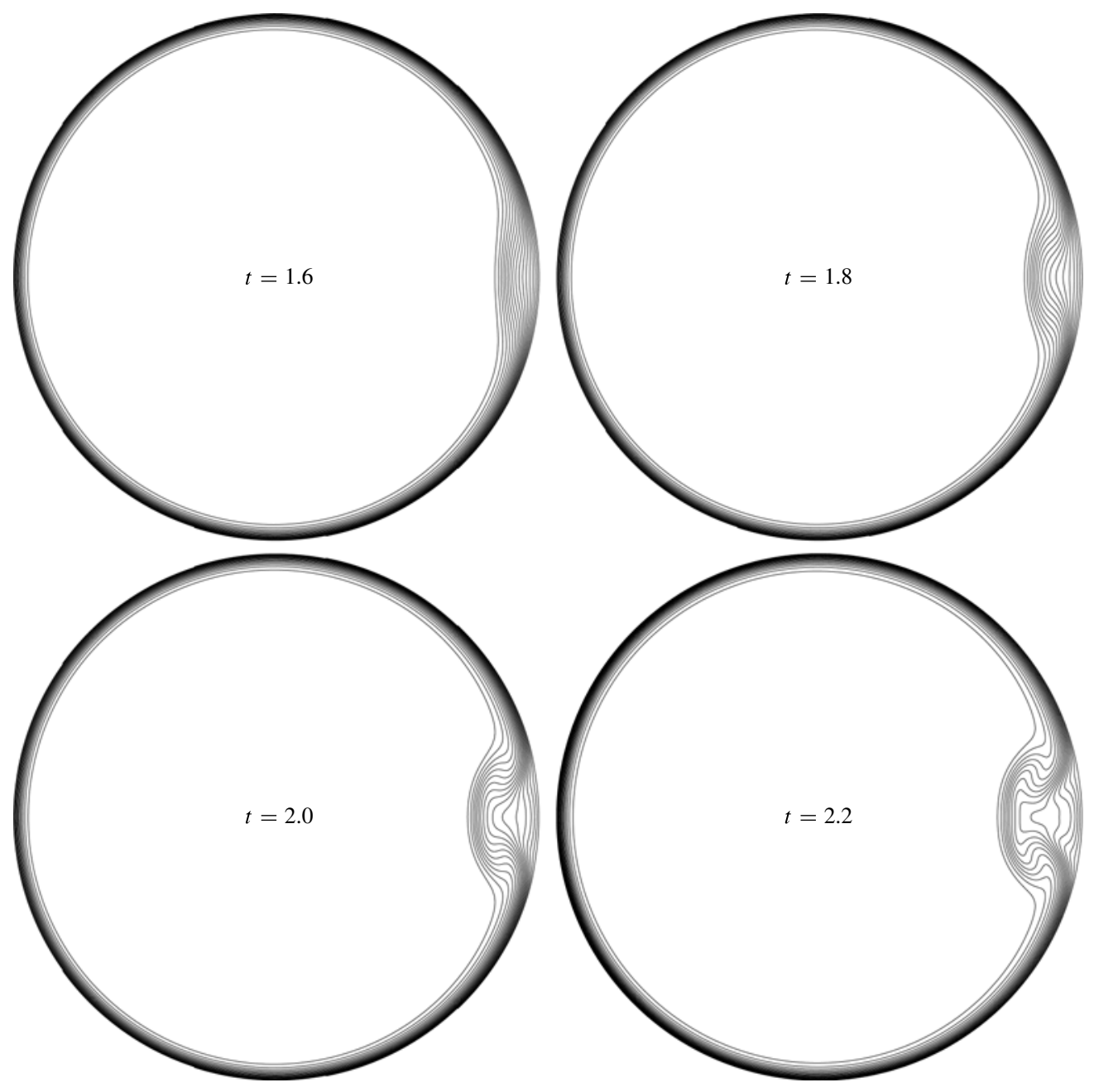

FIGURE 8. Contours of the axial frequency $w(r, \theta, t) /\left(\delta^{-1}+r \cos \theta\right)$ in the meridional crosssection for $t=1.6,1.8,2,2.2$ with $\delta=0.128, E k=1 / 2000$ and $\Omega_{r}=0$ (spin-up from rest). The axis of rotation is to the left of each cross-section and the eruption at the outer bend $(\theta=0)$ is clearly seen; elsewhere in the torus the boundary layer remains quasi-steady over this time scale. Sixteen contours are shown, at values equally spaced between 0.05 and 1 .

generic feature for all $\Omega_{r}$ and play an important role in the response of the flow to non-axisymmetric wave-like instabilities studied in the next section.

\section{Non-axisymmetric instability of the unsteady axisymmetric base flow}

In this section, we first present an asymptotic description of the linear instability of the boundary layer that we believe captures the dominant physical mechanism (the inflectional profiles near breakdown). We then compare these results to the (limited) available experimental data and a (more extensive) sequence of Navier-Stokes simulations.

The experimental investigations of MM and del Pino et al. (2008) have presented evidence of an instability that breaks the rotational symmetry of the flow around the 


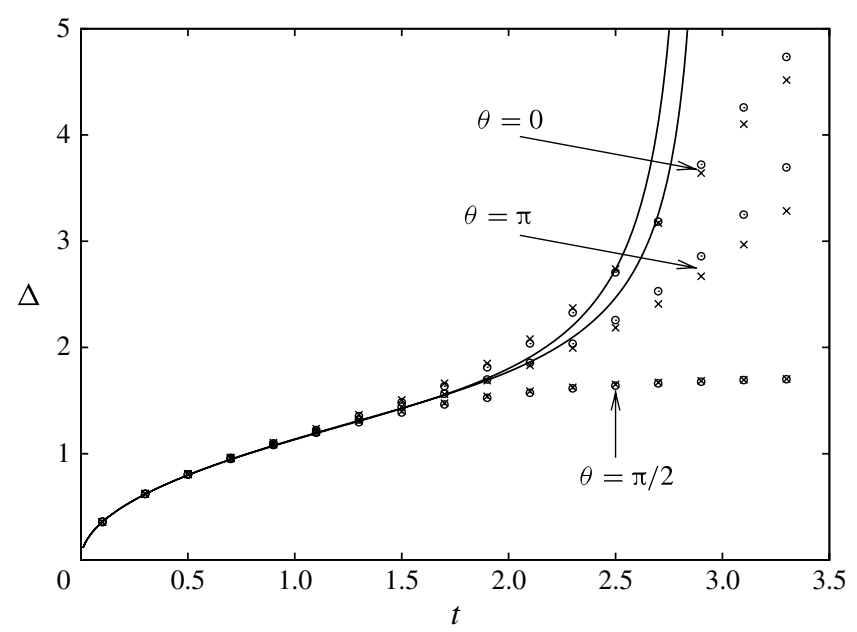

FIGURE 9. The evolution of the boundary-layer thickness $\Delta(\theta, t)$, as defined in (3.13), at the positions $\theta=0, \pi / 2, \pi$ for a case of spin-over with $\Omega_{r}=-0.355$. The solid lines are solutions of the boundary-layer system at $\theta=\pi$ (lower solid line) and $\theta=0$ (upper solid line) whilst the data points are Navier-Stokes computations with $E k=1 / 1000$ (cross), 1/2000 (circle). The curvature is taken to be $\delta=0.128$.
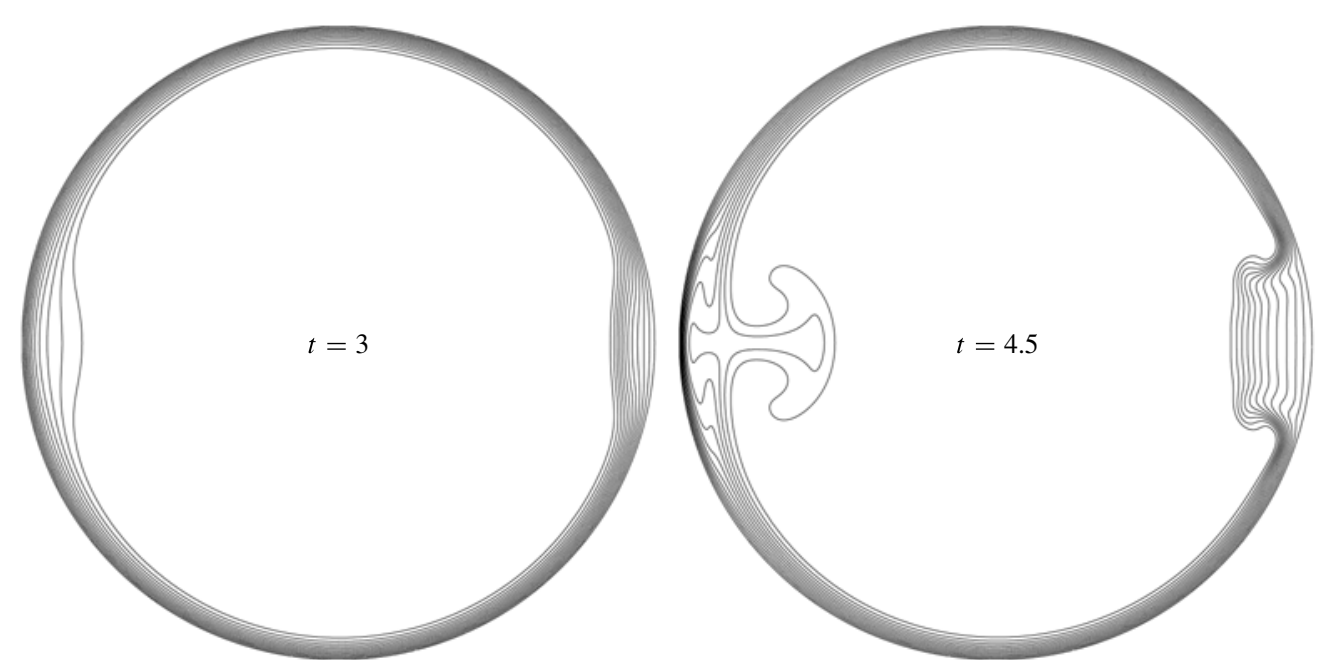

FIGURE 10. Contours of the axial frequency $w(r, \theta, t) /\left(\delta^{-1}+r \cos \theta\right)$ in the meridional crosssection for $t=3,4.5$ with $\delta=0.128, E k=1 / 2000$ and $\Omega_{r}=-0.355$. The axis of rotation is to the left of each cross-section and the simultaneous eruption at both $\theta=0, \pi$ is clearly seen. Twelve equally spaced contours are used between 1 and -0.35 .

torus. The instability occurs within a few rotations of the container, typically at times comparable to those at which the boundary layer is predicted to erupt. Experimental photographs using a light sheet across the equatorial plane of the torus indicate that the instability is wave-like around the torus, see figure 19 of MM, and the wavelength is short. The wave is first observed in the vicinity of the boundary-layer eruption, becomes visible suddenly and usually grows until the flow becomes turbulent. 

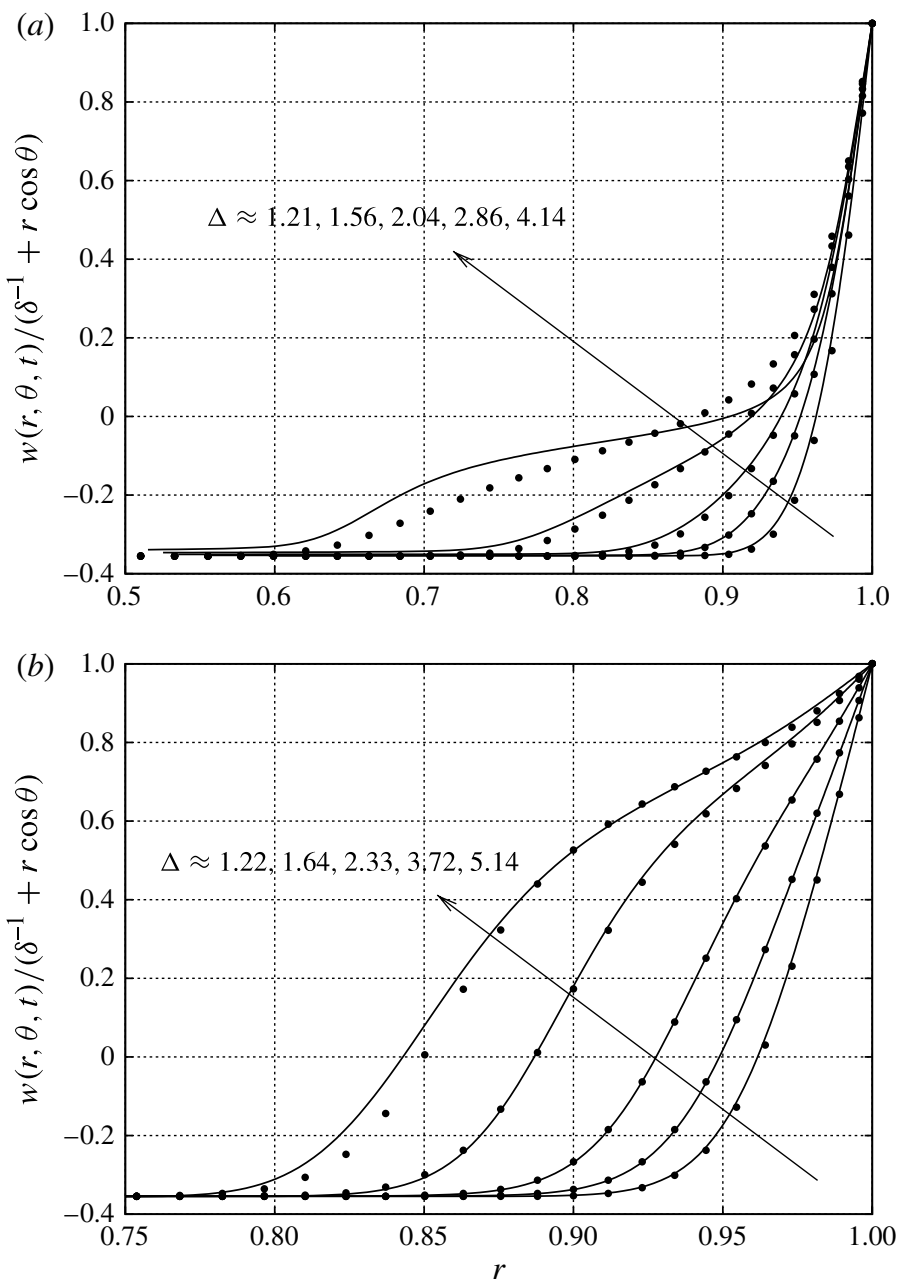

FIGURE 11. The evolution of the axial frequency during eruption of the boundary layer for a case of spin-over with $\Omega_{r}=-0.355$. The lines show the profiles obtained from a Navier-Stokes computation at $E k=1 / 1000$ for $\theta=\pi(a)$ and $\theta=0(b)$; the torus wall is at $r=1$. The data points show the corresponding boundary-layer results when mapped to the $r-\theta$ domain at the chosen value of $E k=1 / 1000$. The curvature is taken to be $\delta=0.128$.

\subsection{An asymptotic description of linear, non-axisymmetric, instability waves}

The short wavelength and extremely rapid development of the instability suggests that, for $E k \ll 1$, it may be possible to describe the mechanism in the context of inviscid perturbation equations. We therefore seek a linear wave-like perturbation of the unsteady boundary-layer flow in the form

$$
\begin{gathered}
u=E k^{1 / 2} u_{0}(\eta, \theta, t)+\epsilon \tilde{u}(\eta) E+\cdots, \\
v=v_{0}(\eta, \theta, t)+\epsilon \tilde{v}(\eta) E+\cdots, \\
w=w_{0}(\eta, \theta, t)+\epsilon \tilde{w}(\eta) E+\cdots, \\
p=p_{0}(\theta)+E k^{1 / 2} p_{1}(\eta, \theta, t)+\epsilon \tilde{p}(\eta) E+\cdots,
\end{gathered}
$$


where $\eta=(1-r) E k^{-1 / 2}$ as before and $\epsilon \ll 1$ is a perturbation amplitude. The shortscale (comparable to the boundary-layer thickness) wave-like component is such that

$$
E=\exp \{\mathrm{i}(\alpha X+\beta Z-\omega \tau)\},
$$

where $X=E k^{-1 / 2} \varrho \phi, Z=E k^{-1 / 2} \theta, \tau=E k^{-1 / 2} t$ and it is to be understood that the real part of the perturbation is taken.

Substitution of (5.1) into the governing equations given in the Appendix leads to Rayleigh's equation at leading order:

$$
\begin{gathered}
\left(\tilde{U}_{B}-\tilde{c}\right)\left(\tilde{\mathrm{D}}^{2}-\tilde{k}^{2}\right) \tilde{u}(\eta)-\tilde{u}(\eta) \tilde{\mathrm{D}}^{2} \tilde{U}_{B}=0, \\
\tilde{k}^{2}=\alpha^{2}+\beta^{2},
\end{gathered}
$$

where $\tilde{\mathrm{D}} \equiv \mathrm{d} / \mathrm{d} \eta, \tilde{c}=\omega / \tilde{k}$ and $\tilde{U}_{B}$ is the base flow velocity resolved in the direction of the phase velocity of the wave:

$$
\tilde{U}_{B}(\eta ; \theta, t)=\frac{\alpha w_{0}+\beta v_{0}}{\tilde{k}} .
$$

The neglected terms in (5.2) are of $O\left(E k^{1 / 2}\right)$, which is simply $O\left(\left(v / \Omega_{f}\right)^{1 / 2} a^{-1}\right)$ a measure of the boundary-layer thickness relative to the radius of the pipe. At these scales, the base flow is effectively steady. However this is only self-consistent in the limit $E k \ll 1$, when waves of wavelength comparable to the boundary-layer thickness grow on a time scale much shorter than the time for one revolution of the torus.

For consistency with the earlier boundary-layer analysis (3.6), we rescale the boundary-layer coordinate $\xi=\varrho^{1 / 4} \eta$, and (5.2) becomes

$$
\begin{gathered}
\left(U_{B}-c\right)\left(\mathrm{D}^{2}-k^{2}\right) \hat{u}(\xi)-\hat{u}(\xi) \mathrm{D}^{2} U_{B}=0, \\
k^{2}=\frac{1}{\varrho^{1 / 2}}\left(\alpha^{2}+\beta^{2}\right),
\end{gathered}
$$

where $\tilde{u}(\eta)=\hat{u}(\xi), \mathrm{D} \equiv \mathrm{d} / \mathrm{d} \xi, c=\tilde{c} / \varrho$ and

$$
U_{B}(\xi ; \theta, t)=\frac{\alpha W+\beta \varrho^{-1 / 2} \sin \theta \Psi_{\xi}}{\left(\alpha^{2}+\beta^{2}\right)^{1 / 2}},
$$

where $W$ and $\Psi$ are defined in (3.5).

The experimental results suggest that an instability wave first occurs at a 'front' that develops near $\theta=0$ when $\Omega_{r}=0$ (spin-up from rest). For $\theta=0$ the base flow has no component in the $\theta$ direction, which motivates examination of the case $\beta=0$ rather than the more general spiral modes $(\beta \neq 0)$; the extension of the analysis in the latter case is straightforward.

As with all such Rayleigh analyses, the development of inflectional profiles is a key feature. In figure 12 we show the location of inflection points $\xi_{c}$ (points at which $\left.W_{\xi \xi}\left(\xi_{c}, \theta, t\right)=0\right)$ in the axial base flow as the boundary-layer solution evolves with time in the two cases: $\Omega_{r}=0, \theta=0$ (spin-up) and $\Omega_{r}=2, \theta=\pi$ (spin-down). In both cases, inflectional profiles develop prior to the boundary-layer eruption, as noted in $\S 4$, and the singular thickening of the boundary layer means that $\xi_{c} \rightarrow \infty$ as $t \rightarrow t_{s}$.

For fixed values of $t$ beyond the point at which inflection points develop, we solve the Rayleigh problem (5.3) in a standard manner, employing a QZ algorithm and local refinement following discretisation using a second-order finite-difference scheme. When $\beta=0$, we find a band of unstable axial wavenumbers $k$. In the spin-up case $\left(\Omega_{r}=0\right)$, the base flow is chosen local to the eruption point at $\theta=0$, whereas for spin-down $\left(\Omega_{r}=2\right)$, the analysis is performed at $\theta=\pi$. Figure 13(a) presents the 

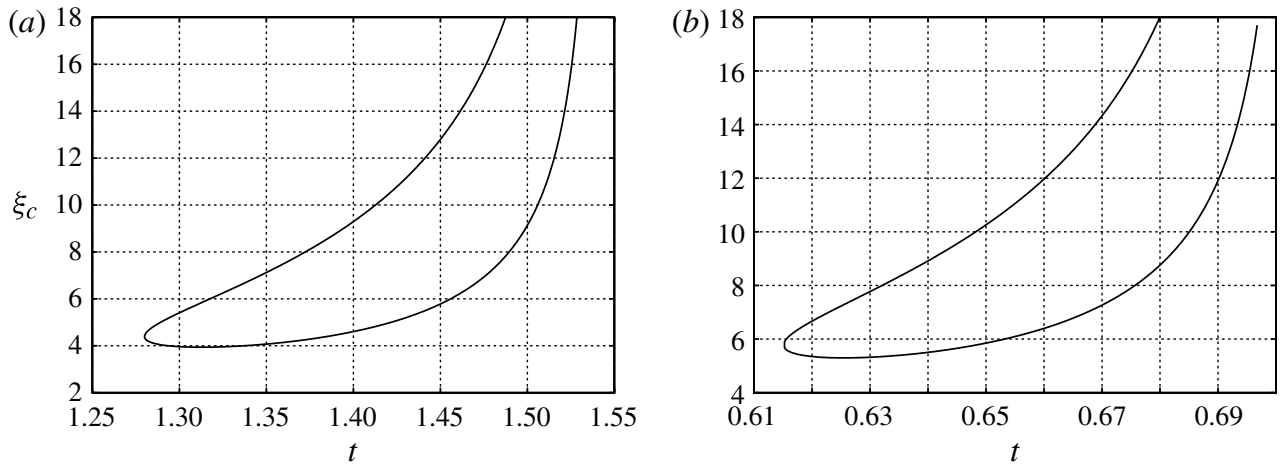

FIGURE 12. The development of inflectional axial flow profiles at the outer bend during spinup $(a)$, and the inner bend during spin-down $(b)$. Here $\xi_{c}$ is the boundary-layer coordinate at which $W_{\xi \xi}=0$. The curvature is taken to be $\delta=0.128$. In both figures the abscissa extends to the time of the singularity, which is at $t \approx 1.55$ in $(a)$ and $t \approx 0.7$ in $(b)$ : (a) $\theta=0 ; \Omega_{r}=0 ;(b) \theta=\pi ; \Omega_{r}=2$.
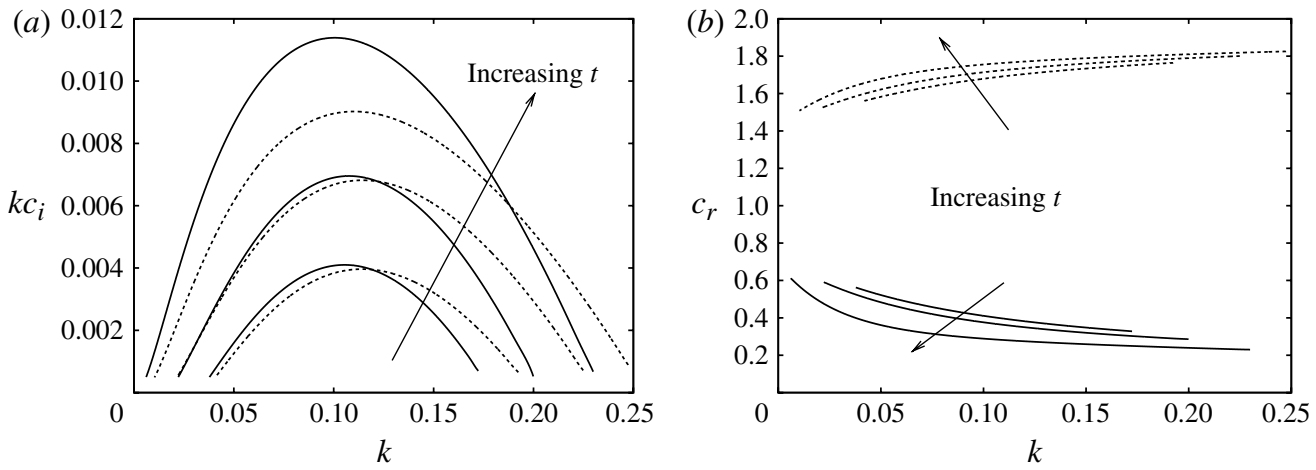

FIgURE 13. (a) The scaled growth rate, $k c_{i}$ as a function of the wavenumber $k$, for the inviscid instability with $\beta=0$. (b) The corresponding phase speed $c_{r}$ as a function of wavenumber $k$ for the data set. In both $(a)$ and $(b)$, two cases are shown: $\Omega_{r}=0, \theta=0$, $t=1.35,1.4,1.5$ (solid lines) and $\Omega_{r}=2, \theta=\pi, t=0.64,0.66,0.68$ (dashed lines).

scaled temporal growth rate $k c_{i}$ (where $c=c_{r}+\mathrm{i} c_{i}$ ) as a function of the wavenumber $k$ for $\Omega_{r}=0,2$ at three different values of $t$ for which the inflection points have developed in the local base flow. In all cases, the maximum growth rate lies in the range $k \approx 0.1$ to $k \approx 0.12$ and the corresponding dimensional growth rate of the wave is $\varrho^{5 / 4} E k^{-1 / 2} k c_{i} \Omega_{f}^{-1}$. Figure $13(b)$ presents the corresponding phase speed $c_{r}$.

\subsection{Experimental work of Madden \& Mullin (1994)}

In the experiments of MM, the Reynolds number corresponding to their figure 19 was $C=7540$ (in their notation) and the curvature parameter was $\delta=0.128$. The corresponding Ekman number is $E k=(\delta C)^{-1} \approx 10^{-3}$ and at the outer wall $(\theta=0)$, $\varrho=\delta^{-1}+\cos \theta=8.8125$. For the fastest growing linear mode, in the $E k \ll 1$ limit when $\Omega_{r}=0$, the predicted number of waves around the torus is $n=k \varrho^{5 / 4} E k^{-1 / 2}$, where $k \approx 0.1-0.11$ (a weakly varying function of the slower time scale over which the base flow varies). Thus, the inviscid local analysis leads to an axial (around 
the torus) wavenumber of $n \approx 50$ for the fastest growing mode (taking $k \approx 0.105$ ). Extrapolation of the section of torus shown in figure 19 of MM leads to the estimate that $n \approx 60$ in the experimental work. This inflectional mechanism is therefore certainly a plausible explanation for the waves observed in the work of MM, but we can make a more detailed comparison by extending our numerical Navier-Stokes solutions to allow for linearised non-axisymmetric modes.

\subsection{Non-axisymmetric stability determined from Navier-Stokes computations}

The asymptotic analysis that we have presented thus far is entirely local to the breakdown position (that is, at the inner or outer bend of the pipe/torus) and is only valid in the low-Ekman-number limit.

At finite values of the Ekman number, the separation of time scales between the instability growth and the base flow development cannot be justified. In such a regime we must consider the growth of any instability in parallel with the base flow evolution.

We assume that the response of the flow is decomposed in the form

$$
\begin{gathered}
\boldsymbol{u}(r, \theta, t)=\boldsymbol{u}_{0}(r, \theta, t)+\epsilon \hat{\boldsymbol{u}}(r, \theta, t) \mathrm{e}^{\mathrm{i} n \phi}, \\
p(r, \theta, t)=p_{0}(r, \theta, t)+\epsilon \hat{p}(r, \theta, t) \mathrm{e}^{\mathrm{i} n \phi},
\end{gathered}
$$

where $\epsilon \ll 1, n$ is a wavenumber, $\hat{\boldsymbol{u}}=(\hat{u}, \hat{v}, \hat{w})$ is the (complex) disturbance velocity field and $\hat{p}$ is the (complex) disturbance pressure field. The formulation and solution of the base flow $\boldsymbol{u}_{0}, p_{0}$ is as described in $\S 4$, whilst the resulting linearised system for the (complex) quantities $\hat{\boldsymbol{u}}, \hat{p}$ can be time marched in an analogous manner for any chosen value of $n$. The computational domain for the disturbance remains the meridional cross-section of the pipe, but a separate finite-element mesh is used to discretise the linearised system of equations. Any quantities required from the base flow are obtained via its finite-element representation and the appropriate correspondence schemes are determined automatically by standard functions within oomph-lib. The use of a separate mesh allows for different patterns of spatial adaptivity in the base flow and disturbance, reflecting the different spatial scales within the two flows. The disturbance flow is discretised using the complex analogue of $Q_{2} P_{1}$ elements, in which the real and imaginary parts of the disturbance fluid velocities are interpolated quadratically and the real and imaginary parts of the disturbance pressures are interpolated linearly. The time-derivative terms in the perturbation equations are again treated implicitly using a second-order BDF2 method and the resulting discrete linear system is solved iteratively using GMRES and a diagonally perturbed ILU preconditioner provided by the Trilinos project, see Heroux et al. (2005). The approximate error in each element is calculated using an estimate based on the recovery of both the real and imaginary parts of the velocity gradients and adaptive refinement is employed as described for the base flow. In general, the maximum number of degrees of freedom in the combined problem was of the order of $5 \times 10^{5}$ and typical time steps were $\mathrm{d} t=0.0025$; these smaller time steps are required to capture the oscillatory (axially propagating) nature of the perturbation field.

To determine the stability of this unsteady flow to the non-axisymmetric mode of wavenumber $n$ we first consider a global kinetic energy measure for the perturbation

$$
\bar{E}=\int_{r=0}^{r=1} \int_{\theta=0}^{\theta=2 \pi} \hat{\boldsymbol{u}} \cdot \hat{\boldsymbol{u}} r \mathrm{~d} \theta \mathrm{d} r,
$$



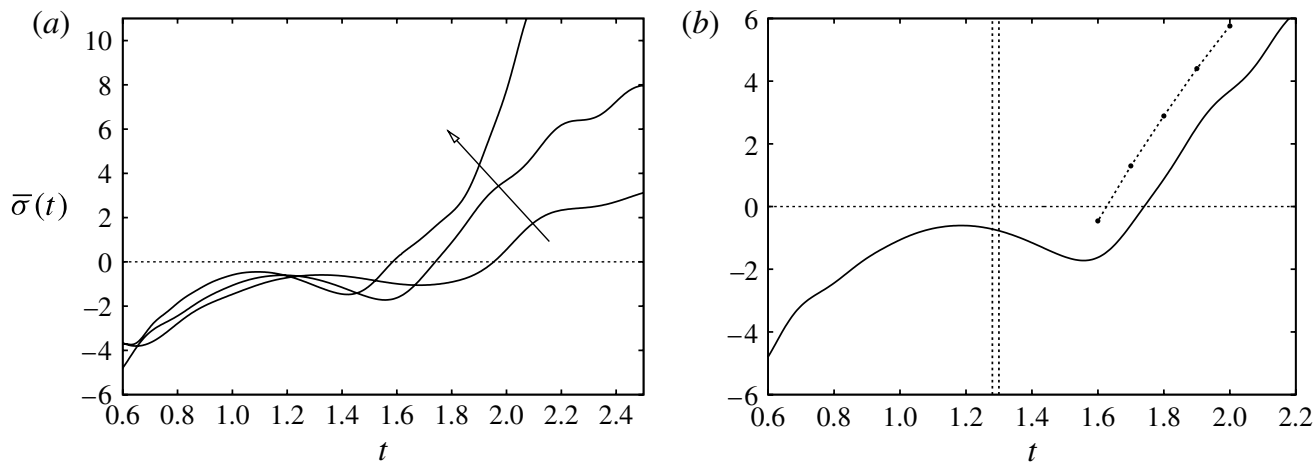

FIgURE 14. (a) The temporal growth rate for non-axisymmetric linear perturbations at $\Omega_{r}=0$ and $E k=1 / 1000,1 / 2000,1 / 4000$ for around-torus wavenumbers of $n=50,71$ and 100 respectively (in the direction of the arrow shown). These wavenumbers are determined from the asymptotic prediction of the fastest growing mode: $n=k \varrho^{5 / 4} E k^{-1 / 2}$ where $k \approx 0.105$, $\varrho=1 / \delta+1$ and $\delta=0.128$. (b) The $E k=1 / 2000$ case is shown again, together with the two-dimensional frozen-time eigenvalue analysis, shown as the data points. The vertical lines indicate the first appearance of an inflection point in the axial flow in the boundary-layer theory $(t \approx 1.28)$ and in the finite- $E k$ calculation $(t \approx 1.3)$.

and then define a growth rate by considering the relative growth,

$$
\bar{\sigma}(t)=\frac{1}{2} \frac{\bar{E}_{t}}{\bar{E}} .
$$

Hence $\bar{\sigma}(t)>0$ corresponds to energy growth of the disturbance, and this definition is also consistent with a modal (linearised) eigenvalue analysis if the base flow is assumed to be steady.

Our aim here is to demonstrate that the local, small-Ekman-number, inviscid asymptotic description of the instability does play a role when applied at small (but finite) Ekman numbers in the two-dimensional cross-section of the finite-radius pipe. In this context we choose to investigate a simplistic ad-hoc initiation mechanism for the non-axisymmetric mode, by imposing boundary conditions of

$$
\begin{gathered}
\hat{u}(r=1, \theta, t)=0, \quad \hat{v}(r=1, \theta, t)=0, \\
\hat{w}(r=1, \theta, t)=\varrho \theta \exp \left[-a_{1} \theta^{2}\right] \exp \left[-a_{2}\left(t-t_{k}\right)^{2}\right],
\end{gathered}
$$

where $a_{1}=100, a_{2}=250$ and $t_{k}=0.25$. The boundary condition for $\hat{w}$ is chosen to be spatially localised near the outer bend of the torus to reduce the number of degrees of freedom in the discretised linear perturbation equations, whilst the transient behaviour in time ensures that the linearised system is unforced beyond $t \approx 0.5$. We have confirmed that the exact nature of the transient perturbation does not influence the qualitative aspects of the results we present below but does lead to instability at slightly different values of $t$ at finite values of $E k$.

In figure 14(a) we show the growth rate $\bar{\sigma}(t)$ for $\Omega_{r}=0$ with $E k=1 / 1000,1 / 2000$, $1 / 4000$ and correspondingly increasing wavenumbers $n=50,71$ and 100 respectively. These choices of $n$ are based on the predictions of the previous asymptotic theory, which suggests an around-torus wavenumber $n=k \varrho^{5 / 4} E k^{-1 / 2}$, where $k \approx 0.105$. The linearised inviscid analysis coupled with the unsteady boundary-layer evolution predicts that instability occurs at the first appearance of an inflection point in the axial flow at $\theta=0$, which is at $t \approx 1.3$ (as shown in figure 12). As previously discussed, 
at finite Ekman numbers the development of inflectional profiles is delayed and the results presented in figure 14(a) confirm that the instability develops when $t>1.3$, but that the time at which the instability occurs decreases with the Ekman number.

Instead of time marching the perturbation equations we can also determine the growth rate of the most unstable non-axisymmetric eigenmode directly from the linearised perturbation equations by solving a two-dimensional eigenproblem for the growth rate $\lambda$, under the assumption that the temporal dependence of all perturbation quantities is $\mathrm{e}^{\lambda t}$. Such a 'frozen-time' analysis is non-rigorous except in the limit of $E k \rightarrow 0$ and provides a temporal growth rate parameterized by time $t$. Figure 14(b) shows the same unsteady data from figure 14(a) in the case of $\Omega_{r}=0, E k=1 / 2000$, $n=71$, but together with the growth rate predicted by the frozen-time analysis. Both the unsteady marching and the frozen-time analysis present consistent pictures of the temporal growth of the non-axisymmetric instability, although the frozen-time analysis predicts instability at a slightly earlier time.

The absolute value of the perturbation's axial flow component, $|\hat{w}(r, \theta, t)|$, as obtained from the time marching process, is shown in figure 15 for the same case of $E k=1 / 2000, \Omega_{r}=0, n=71$. These views of the disturbance field are presented at the same times as the corresponding base flows shown in figure 8. In figure 16 we show the same data, but for a cross-section taken across the mid-plane of the pipe, with the (linear) disturbance field's axial component normalized to have a maximum value of unity. Figure 16(a) shows the inflection point as it propagates inwards during the eruption process at the outer bend, whilst the inner region is entirely benign and quasi-steady. Consistent with the local $E k \ll 1$ analysis, we see from figure 16(b) that the unsteady disturbance is concentrated near the outer inflection point of the base flow profile. We conclude that the experimentally observed instability is, indeed, inviscid in origin.

\section{Centrifugal axisymmetric instability}

In addition to the inviscid, non-axisymmetric instability described above, the system is also susceptible to an axisymmetric centrifugal instability when $t \ll 1$. The boundary layer is developing in time, which means that the problem shares many features with the Görtler vortex problem, as reviewed by Hall (1990).

As described in $\S 3.1$, the initial response of the flow to a change in the rotation rate of the torus is a velocity field of the form

$$
\boldsymbol{u}=\left(E k^{1 / 2} t^{3 / 2} U_{B}(\hat{Y}, \theta), t V_{B}(\hat{Y}, \theta), W_{B}(\hat{Y}, \theta)\right)+\cdots,
$$

in a Rayleigh layer defined by $r=1-\hat{Y} E k^{1 / 2} t^{1 / 2}$ adjacent to the toroidal wall. In terms of the previous solution given above as (3.9):

$$
\begin{gathered}
W_{B}(\hat{Y}, \theta)=\varrho(\theta) \hat{W}(\hat{Y}), \\
V_{B}(\hat{Y}, \theta)=\varrho(\theta) \sin \theta \hat{\Psi}_{\hat{Y}}(\hat{Y}),
\end{gathered}
$$

where, as defined earlier, $\varrho(\theta)=\delta^{-1}+\cos \theta$. The radial velocity component, $U_{B}$, is not given explicitly because it plays no role in the perturbation equations (6.9).

For a balance that is able to support a centrifugal instability mode (at $O(1)$ values of the curvature parameter $\delta$ ), with a perturbation velocity field $\left(u_{p}, v_{p}, w_{p}\right)$, we require $\partial_{\theta} \sim \partial_{r}$ to conserve mass, $\partial_{t} \sim E k \partial_{r} \partial_{r}$ to maintain viscous diffusion across the boundary layer, and $\partial_{t} u_{p} \sim w_{p} W_{B}$ and $\partial_{t} w_{p} \sim u_{p} \partial_{r} W_{B}$ to maintain the centrifugal 


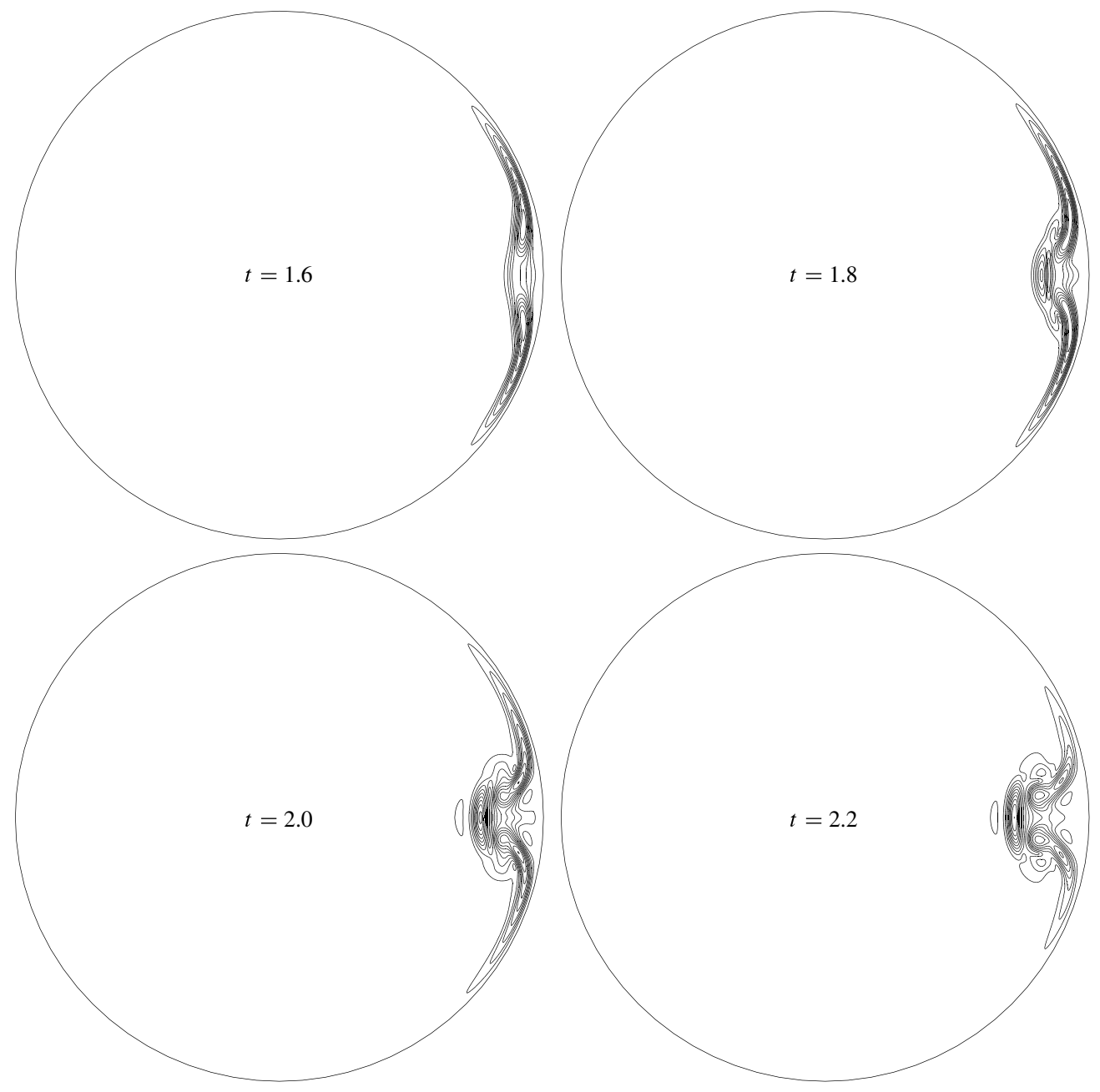

FIgURE 15. Contours of the absolute value of the axial flow component, $|\hat{w}(r, \theta, t)|$, of the wave-like (non-axisymmetric) perturbation; see (5.4). These cross-sections are for $E k=1 / 2000, \Omega_{r}=0, \delta=0.128, n=71$ and $t=1.6,1.8,2$ and 2.2. The axial component of the base flow is as shown in figure 8 . Ten contours (equally spaced) are shown in each crosssection; the ranges are [0.005, 0.0514], [0.005, 0.0416], [0.02, 0.0711] and [0.02, 0.233] as $t$ increases.

forcing. Such a balance is seen to be achieved over a short time/space scale defined by

$$
\begin{gathered}
T=E k^{-1 / 3} t, \\
Y=(1-r) E k^{-2 / 3}, \\
\Theta=\theta E k^{-2 / 3},
\end{gathered}
$$

with $u_{p}, v_{p} \sim w_{p} E k^{1 / 3}$. These time/length scales have also been obtained for the onedimensional flow induced in the Rayleigh layer on an impulsively rotated infinite cylinder by Otto (1993) and Mackerrell, Blennerhassett \& Bassom (2002). We seek a multi-scale solution in which the base flow varies around the torus on the slow scale $\theta$ but the perturbation depends on the fast scale $\Theta$. The velocity field for the base flow 

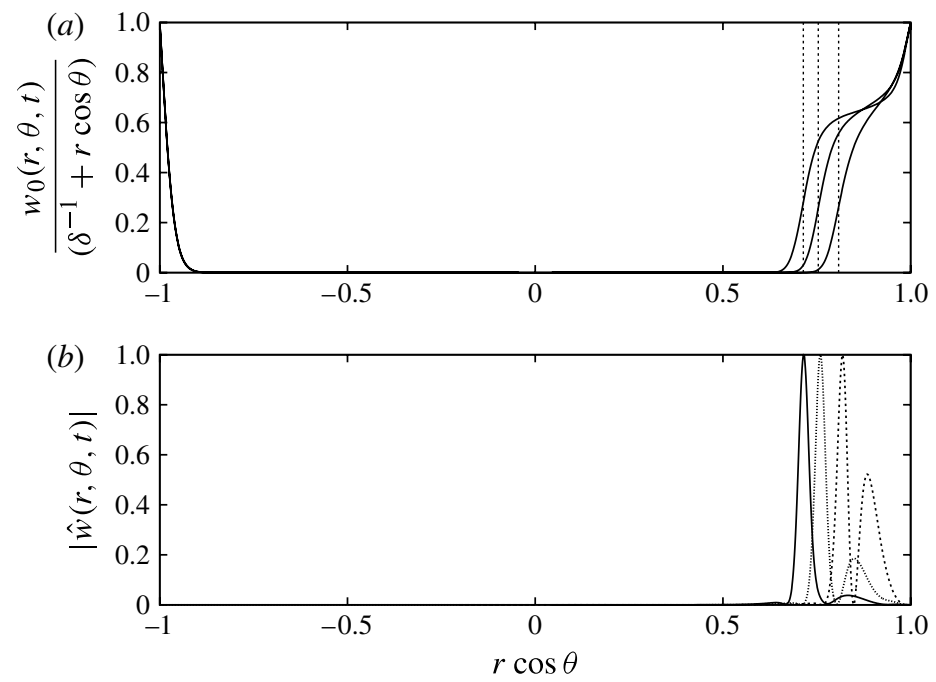

FIGURE 16. Cross-sections across the mid-plane $(\theta=0, \pi)$ of $(a)$ the base-flow axial frequency (as seen in figure 8) and $(b)$ the magnitude of the (complex) perturbation axial component (as seen in figure 15). These data are presented for the same parameters $\left(E k=1 / 2000, \Omega_{r}=0, \delta=0.128, n=71\right)$ and for $t=1.8,2,2.2$, which are shown as dashed, dotted and solid lines respectively in $(b)$. The vertical lines in $(a)$ indicate the outermost inflection point in the base axial flow and the perturbation has been normalized such that the peak value is unity.

plus perturbation is therefore of the form

$$
\begin{aligned}
\boldsymbol{u}= & \left(E k T^{3 / 2} U_{B}(\hat{Y}, \theta), E k^{1 / 3} T V_{B}(\hat{Y}, \theta), W_{B}(\hat{Y}, \theta)\right) \\
& +\epsilon\left(E k^{1 / 3} \tilde{u}(Y, \Theta, T), E k^{1 / 3} \tilde{v}(Y, \Theta, T), \tilde{w}(Y, \Theta, T)\right)+\cdots
\end{aligned}
$$

with a pressure perturbation of the form $\tilde{p}(Y, \Theta, T) E k^{2 / 3}$, required to maintain the pressure gradient in the linearised perturbation equations. In (6.7) $\epsilon$ is a measure of the Taylor-Görtler perturbation and we assume that it is sufficiently small to yield a purely linear problem.

For a Fourier mode with wavenumber $K$ over the short scale $\Theta$ we decompose the perturbation as

$$
(\tilde{u}(Y, \Theta, T), \tilde{v}(Y, \Theta, T), \tilde{w}(Y, \Theta, T))=(\hat{u}(Y, T), \hat{v}(Y, T), \hat{w}(Y, T)) \mathrm{e}^{\mathrm{i} K \Theta}+\text { c.c. }(6.8
$$

Clearly, the linear perturbation is also implicitly a function of the slow scale variable $\theta$ (via the base flow variation), but we will ignore this dependence and perform a purely local analysis under the assumption that $E k \ll 1$. The unsteady evolution equations for the perturbation Fourier mode are:

$$
\begin{gathered}
\mathscr{D}^{2} \hat{u}=\hat{g} \\
\left(\mathscr{D}^{2}-\frac{\partial}{\partial T}\right) \hat{g}=2 K^{2} \frac{\cos \theta}{\varrho(\theta)} W_{B} \hat{w}+2 \mathrm{i} K \frac{\sin \theta}{\varrho(\theta)} \frac{\partial}{\partial Y}\left(W_{B} \hat{w}\right)+T \mathrm{i} K\left(V_{B} \mathscr{D}^{2} \hat{u}-\frac{\partial^{2} V_{B}}{\partial Y^{2}} \hat{u}\right) \\
\left(\mathscr{D}^{2}-\frac{\partial}{\partial T}\right) \hat{w}=-\frac{\partial W_{B}}{\partial Y} \hat{u}+T \mathrm{i} K V_{B} \hat{w}
\end{gathered}
$$


where the differential operator $\mathscr{D}$ is defined by

$$
\mathscr{D}^{2} \equiv \frac{\partial^{2}}{\partial Y^{2}}-K^{2}
$$

and the base flow velocities are on the Rayleigh scale $\hat{Y}=Y T^{-1 / 2}$.

As is typical, see Denier, Hall \& Seddougui (1991) and Otto (1993) for example, we consider the receptivity of these centrifugal modes to a wall-roughness forcing mechanism. However, it should be noted that other mechanisms can exist in any experimental configuration; for example, if the initial state is rigid-body rotation, it may be arrived at by the slow decay of transient turbulent flow or inertial waves in the core. For a Fourier component of the wall roughness defined by the boundary $r=1-\epsilon E k^{2 / 3} \mathrm{e}^{\mathrm{i} K \Theta}+$ c.c the boundary conditions for the perturbation follow from linearisation of the no-slip condition onto the unperturbed boundary

$$
\hat{u}=0, \quad \hat{u}_{Y}=-T \mathrm{i} K \frac{\partial V_{B}}{\partial Y}, \quad \hat{w}=-\frac{\partial W_{B}}{\partial Y}, \quad \text { on } Y=0
$$

and

$$
\hat{u}=\hat{u}_{Y}=\hat{w}=0, \quad \text { as } Y \rightarrow \infty .
$$

As the base flow is developing on the thickening Rayleigh-scale coordinate $\hat{Y}$ it is sensible to use the same coordinate in the perturbation equations. Similarly we build in the small- $T$ asymptotic structure of the perturbation by defining

$$
\hat{u}(Y, T)=\bar{u}(\hat{Y}, T) T, \quad \hat{g}(Y, T)=\bar{g}(\hat{Y}, T), \quad \hat{w}(Y, T)=\bar{w}(\hat{Y}, T) T^{-1 / 2},
$$

where these scalings are determined by the wall-roughness forcing. After substitution of (6.11) into (6.9) we solve the resulting system for the quantities $\bar{u}, \bar{g}$ and $\bar{w}$ as a function of the rescaled time $T$ by a standard second-order finite-difference scheme. It is known from, for example, Hall (1985) and Zurigat \& Malik (1995) that crossflow acts to inhibit Taylor-Görtler vortices, so we concentrate on the two regions $\theta=0, \pi$ at which the crossflow velocity $V_{B}$ vanishes.

At any given time we determine the leading-order contribution to the kinetic energy of the perturbation:

$$
E=\int_{Y=0}^{\infty}|\hat{w}|^{2} \mathrm{~d} Y=T^{-1 / 2} \int_{\hat{Y}=0}^{\infty}|\bar{w}|^{2} \mathrm{~d} \hat{Y},
$$

and then the growth rate of the instability in the developing flow is defined to be $\sigma(T)=E_{T} / E$.

In figure 17 we show (as data points) the functional dependence of $T_{n}$ on the perturbation wavenumber $K$ where $T_{n}$ is defined to be the time of neutral growth with $\sigma\left(T_{n}\right)=0$. Over this small time scale (the dimensional time being $T E k^{1 / 3} \Omega_{f}^{-1}$ ) the base flow is essentially, to leading order, a thickening Rayleigh layer. This $T^{1 / 2}$ thickening of the base-flow boundary layer will ultimately lead to the stabilisation of the instability to any $O\left(E k^{2 / 3}\right)$ wavelengths. However, on a time scale of $T=O\left(E k^{-1 / 3}\right)$ the thickening of the Rayleigh layer saturates and becomes a quasi-steady three-dimensional boundary layer with thickness $O\left(E k^{1 / 2}\right)$. For this later problem it may still be possible to obtain a centrifugal instability by seeking larger wavelength perturbations. We have not investigated this additional problem as it seems likely that the modes we discuss here will become unstable to secondary instabilities before any such (later) regime is reached. 


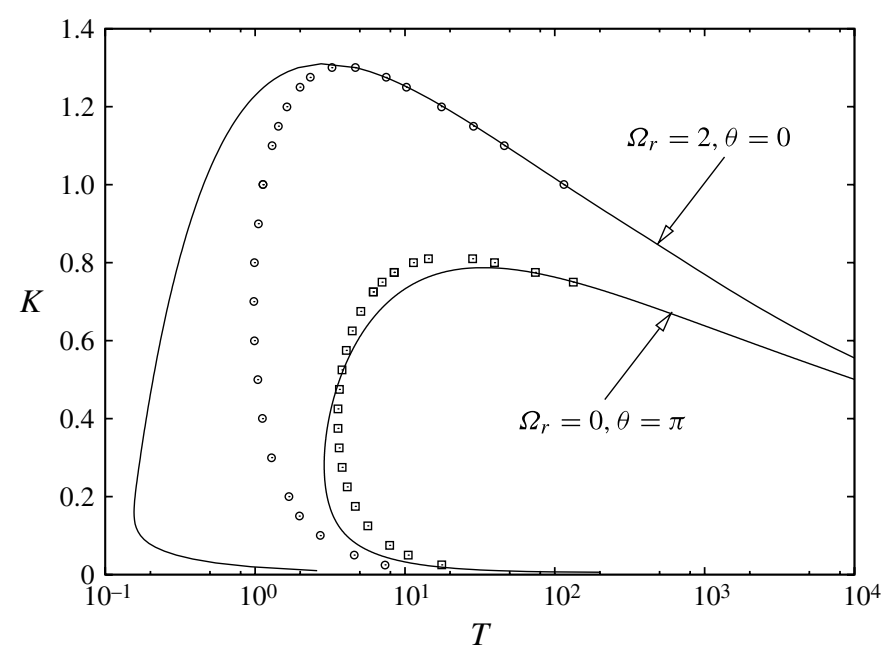

FIGURE 17. The neutral curves for $\Omega_{r}=0$ (spin-up from rest) and $\Omega_{r}=2$ (spin-down). For $\Omega_{r}=0$, instability is obtained at the innermost point of the torus $(\theta=\pi)$ whilst the outermost point remains stable. For $\Omega_{r}=2$, instability is obtained at the outermost point of the torus $(\theta=0)$ whilst the innermost point remains stable. The data points show the time of neutral growth where $\sigma(T)=0$, whilst the solid lines indicate where the frozen-time eigenvalue analysis predicts instability. The curvature is taken to be $\delta=0.128$.

In figure 17 we also show the results of a frozen-time eigenvalue analysis applied to (6.9) for which, rather than solving the initial-value problem, we fix the base flow and obtain an eigenvalue set parameterized by $T$ and $K$. Clearly such an approach is not valid in general, as the time scale over which the base flow develops is the same as the time scale over which the instability grows. However, as is well known, the results of these two approaches agree for the upper-branch modes, for which $K \sim T^{-1 / 8}$, as described by Mackerrell et al. (2002).

As may be anticipated, it is the innermost point of the toroidal container $(\theta=\pi)$ that becomes unstable when the rotation rate is increased $\left(0 \leqslant \Omega_{r}<1\right)$, whilst for spindown $\Omega_{r}>1$ these modes develop at the outermost point $(\theta=0)$. As a consequence, the centrifugal instability is only active (over this small time scale) at the point in the flow that does not (at a later time) develop a finite-time breakdown when $\Omega_{r} \geqslant 0$.

For the more complicated case of spin-over, the instability can occur at both $\theta=0$ and $\theta=\pi$, as is shown in figure 18. It is seen that the instability develops first in the neighbourhood of $\theta=\pi$ and then later at $\theta=0$. Therefore, it is possible that the early development of a centrifugal instability driven by a receptivity mechanism could occur before the boundary layer breaks down with a singular structure.

Figure 19 shows the growth rates determined from solving (6.9) as an initial-value problem for the three cases of $\Omega_{r}=2,0,-0.355$, each with a fixed wavenumber of $K=0.5$. It is clearly seen that the growth rate in the case $\Omega_{r}=2$ is significantly larger (and occurs earlier) than in the other cases and provides the most likely case for investigation in Navier-Stokes computations. Therefore, in the results that follow below, we will restrict attention to $\Omega_{r}=2$.

\subsection{Navier-Stokes simulations with an isolated wall perturbation}

We can demonstrate the existence of this instability in the Navier-Stokes computations by considering a perturbed problem with a wall location defined 


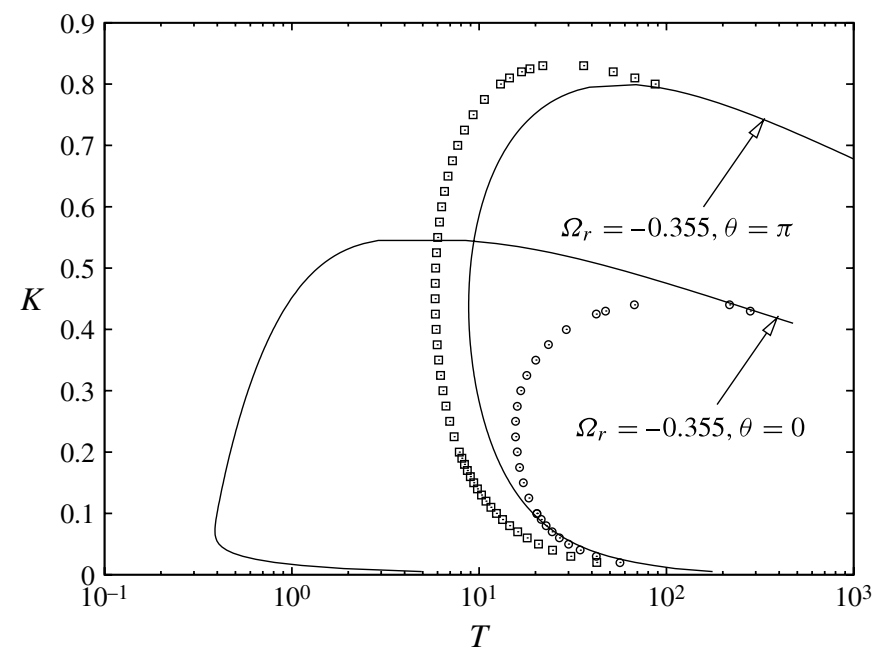

FIGURE 18. The neutral curves for a spin-over case with $\Omega_{r}=-0.355$. In this case centrifugal instability can be found at both the innermost $(\theta=\pi)$ and outermost $(\theta=0)$ positions of the torus. The data points are the positions where $\sigma(T)=0$ whilst the solid lines indicate where the frozen-time eigenvalue analysis predicts instability. The curvature is taken to be $\delta=0.128$.

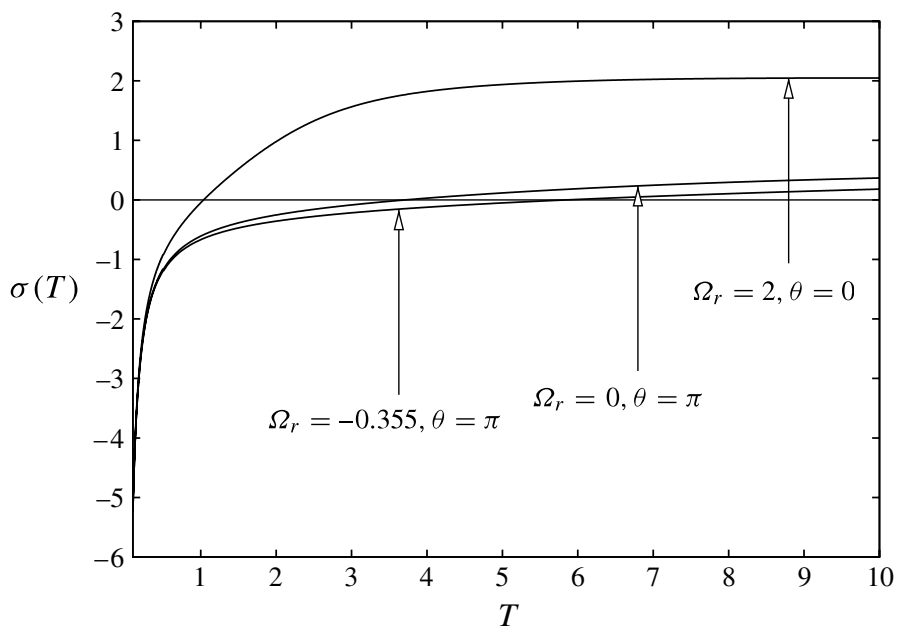

FIGURE 19. The growth rate $\sigma$ as a function of the rescaled time $T$ in the three cases $\Omega=2,0,-0.355$ for a Fourier mode with $K=0.5$. The curvature is taken to be $\delta=0.128$.

by $r_{\text {wall }}=1-\epsilon A E k^{2 / 3} f\left(\theta / E k^{2 / 3}\right)$; that is, rather than solving the system in a circular domain we introduce a small scale perturbation of size $O\left(\epsilon E k^{2 / 3}\right)$ localised about $\theta=0$. We consider a specific case of $E k=1 / 2000, \epsilon=1 / 10$ and $f(\Theta)=\exp \left(-\Theta^{2} / 4\right)$. These values lead to a perturbation to the boundary of the computational domain of a height that is of dimensionless amplitude $6.3 \times 10^{-4} A$ (relative to the radius of the torus). We then perform two computations, one with the boundary perturbation $(A=1)$ and one without $(A=0)$. In each case we can compute the shear at the wall in the 

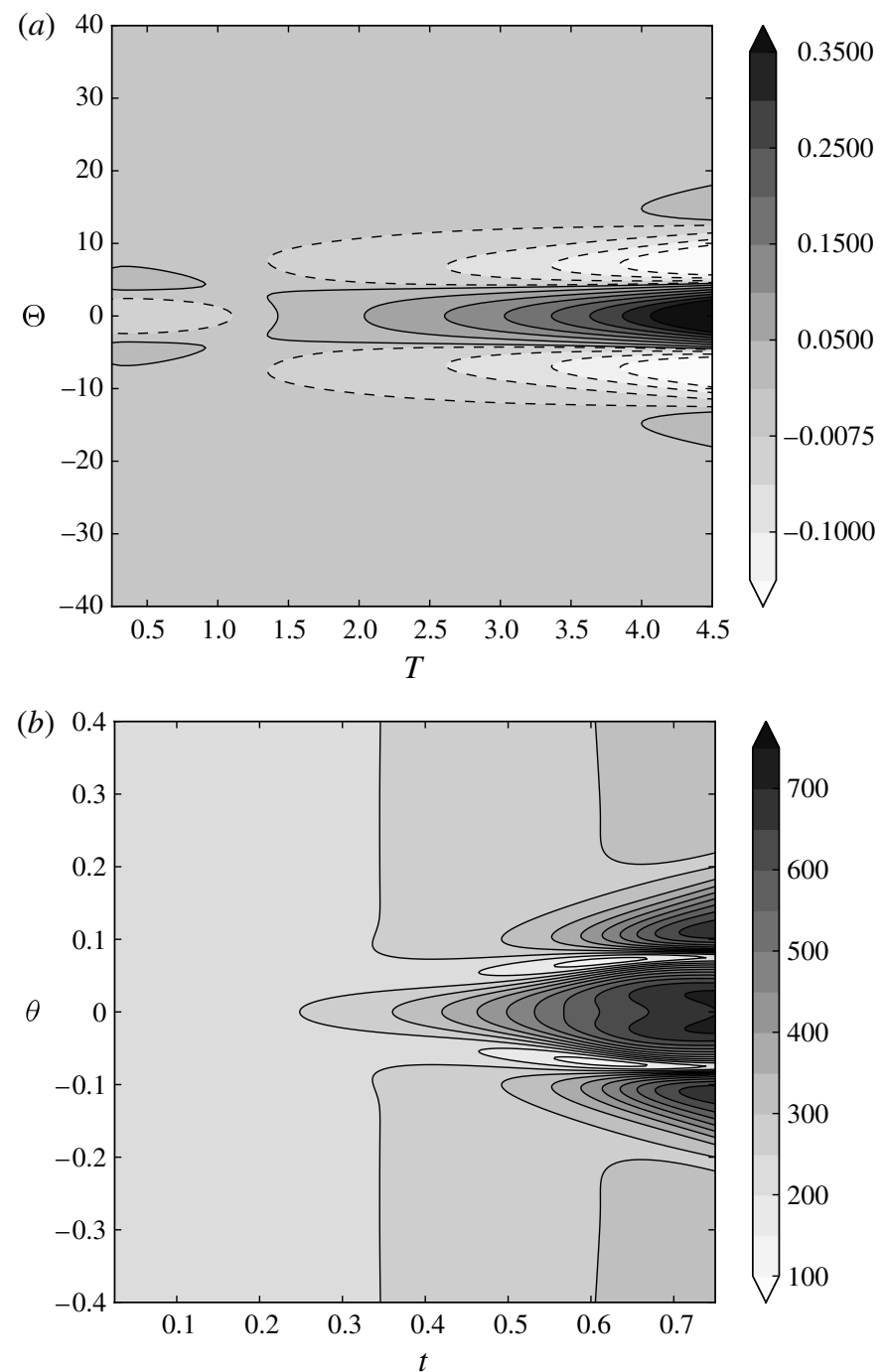

FIGURE 20. Axisymmetric unsteady Navier-Stokes computations, showing the contours of the shear stress $\tau(\theta, t)=\partial w / \partial r$ evaluated at the wall of the torus, near $\theta=0$, in the case $\Omega=2, \delta=0.128$ and $E k=1 / 2000$. (a) The initial evolution of a scaled perturbation $\Delta \tau E k^{2 / 3}$ induced by an imperfection added to the wall position of dimensionless amplitude $6.3 \times 10^{-4}$ (see the text for details). The contours are shown on the time/space scales associated with a centrifugal instability, where $\theta=\Theta E k^{2 / 3}$ and $t=T E k^{1 / 3}$. (b) The evolution of $\tau t^{1 / 2}$ in the unscaled coordinates, demonstrating the nonlinear development of the perturbation over a somewhat longer time scale. Note that the results of figure 17 predict growth of linear perturbations at $T \approx 1$. The curvature is taken to be $\delta=0.128$.

vicinity of the perturbation:

$$
\tau(\theta, t)=\left.\frac{\partial w}{\partial r}\right|_{r=r_{\text {wall }}} .
$$

In figure $20(a)$ we show the difference in these two data sets $\left(\Delta \tau=\left.\tau\right|_{A=1}-\left.\tau\right|_{A=0}\right)$ as a function of $\Theta$ and $T$, including the predicted $E k^{2 / 3}$ scaling for the contribution of the instability to the wall shear. We note that this same computation but at $E k=1 / 1000$ 
displayed very little difference from the case shown, suggesting that the scalings of this section capture the growth mechanism accurately. In figure 20(b) we show contours of the quantity $\tau t^{1 / 2}$ for the $A=1$ case, showing the nonlinear evolution of the Taylor-Görtler vortex in the unscaled $\theta$ and $t$ variables.

Figure 20(a) shows initial decay of the perturbation induced by the $O\left(\epsilon E k^{2 / 3}\right)$ wall imperfection followed by subsequent growth around $T \approx 1$ in line with the boundarylayer predictions of figure 17. Such behaviour is entirely typical of these flows and can be found in, for example, Denier et al. (1991). The difference here is that we are extracting the behaviour from a data set obtained via the nonlinear Navier-Stokes equations, rather than reconstructing Fourier components of the linearised perturbation equations on top of the boundary-layer system. This makes the nonlinear continuation to larger times a simple process, for which figure $20(b)$ demonstrates that the instability has grown to significantly affect the base flow at $t \approx 0.5$ when $E k=1 / 2000$. The finite-time singularity of the boundary-layer system is at $t \approx 0.7$ (albeit at $\theta=\pi$, rather than the $\theta=0$ location considered here). Therefore, at values of the Ekman number typical of laboratory experiments (e.g. $O\left(10^{-3}\right)$ as used by $\mathrm{MM}$ and del Pino et al. 2008) the nonlinear development of a centrifugal instability typically still occurs on the same time scale as the boundary-layer eruption. Only at much lower values of $E k$ would we expect to see significant scale separation between the centrifugal modes which begin to grow when $t=O\left(E k^{1 / 3}\right)$ and the breakdown which occurs for $t=O(1)$. Nevertheless, because the centrifugal instability occurs at $\theta=0$ and the finite-time singularity occurs at $\theta=\pi$ (in the case of $\Omega_{r}=2$, and vice versa if $0 \leqslant \Omega_{r}<1$ ) the two events remain largely isolated until the core flow is altered post breakdown. However, the possibility exists for the two mechanisms to interact in the spin-over $\left(\Omega_{r}<0\right)$ regime, but we do not pursue this here.

\section{Conclusions}

This work is motivated by the experimental investigation of Madden \& Mullin (1994), which considered the transient evolution of the flow in a toroidal container during a rapid increase in rotation rate, from rest to a constant final value. Their results demonstrated that for sufficiently small Reynolds numbers (based on the final rotation frequency) the spin-up response is diffusive whilst at intermediate values the spin-up process is aided by an increasingly strong secondary recirculation. However, for large Reynolds numbers the response is much more complicated with the development of a 'front' that propagates radially inwards from the outermost bend of the container. This front is initially axisymmetric, but rapidly develops a wave-like structure in the around-torus direction, which in turn leads to a transient, disordered, three-dimensional flow.

We believe that we have described the dominant mechanisms in the Madden \& Mullin (1994) experimental configuration through a detailed description of the rotationally symmetric base flow combined with an analysis of its linear stability properties. Furthermore we have extended the analysis to apply to a full range of flows that can be parameterised by the ratio of initial-to-final rotation frequencies, $\Omega_{r}$. Our boundary-layer analysis is combined with large-scale numerical solutions (at finite Reynolds number) of the nonlinear rotationally symmetric flow coupled with the linearised unsteady non-axisymmetric flow field for a Fourier mode in the around-torus direction. We have presented evidence that a collisional boundary layer is indeed the underlying mechanism of the 'front' observed in the experiments, with good agreement being demonstrated between the small-Ekman-number (large- 
Reynolds-number) asymptotics and finite-, but small-, Ekman-number computations of the full flow field.

A crucial observation is that the boundary-layer collision and subsequent eruption leads to axial velocity profiles that are inflectional and we demonstrate that a nonaxisymmetric, inviscid instability does occur in the boundary-layer equations. This mechanism both explains the rapid growth and provides a good estimate for the number of waves observed in the experimental configuration. In addition, timemarching the (linearised) two-dimensional flow field of a Fourier mode in conjunction with a developing unsteady rotationally symmetric base flow at small values of the Ekman number shows good agreement with the (local) boundary-layer analysis and demonstrates that the instability is realised in the full field equations.

Although the instability waves at the 'front' arise from an inflectional instability, the importance of centrifugal modes (as highlighted by $\mathrm{MM}$ ) is also confirmed in our analysis. The inflectional instability is, in essence, governed by a one-dimensional eigenvalue problem because of the separation of time scales obtained for $E k \ll 1$. However, no such eigenvalue problem is available for the centrifugal instability, which can only be rigorously described in the context of an appropriately formulated initialvalue problem. The centrifugal instability develops on an asymptotically short time scale $O\left(E k^{1 / 3} \Omega_{f}^{-1}\right)$ after the transition in the rotation frequency of the container and, provided that $\Omega_{r}>0$, the instability develops diametrically opposite the eruption point in the container. For Ekman numbers typical of experimental values, $E k \approx 10^{-3}$, the time scales for the eruption and for growth of centrifugal modes are not particularly well separated. We have demonstrated that the asymptotic boundary-layer predictions regarding the growth of centrifugal modes caused by wall perturbations can be obtained (and their nonlinear evolution captured) by careful computation of the Navier-Stokes equations at finite values of $E k$. We expect these developing transient vortex states to become unstable to a secondary (non-axisymmetric) instability mechanism; however we have not examined that problem in this work.

A feature of the experiments described by MM that we cannot explain is the observed non-uniqueness of transient evolutions, as revealed by their phase-space reconstruction methods. We can only echo their comments that this non-uniqueness is likely to be associated with the nonlinear (non-axisymmetric) development of the prevailing instability mechanisms. The description of such flows would require a nonlinear three-dimensional unsteady solution of the field equations.

Throughout this work (to avoid the introduction of an additional parameter) we have taken the change in rotation rate to be impulsive; and to facilitate comparison with their work, we chose the curvature parameter, $\delta$, to correspond to that of Madden et al.'s experimental configuration. The qualitative nature of the results that we present is unchanged by other values of $\delta=O(1)$. Moreover, a consequence of our choice of scaling (3.5) is that the results at the equatorial points $\theta=0, \pi$ are only influenced by $\delta$ through a time rescaling. We have verified that our results apply to other monotonic changes in the rotation rate, provided that the time scale over which the transition occurs is sufficiently rapid compared to the rotation period of the container. Indeed, even for transitions on the $O\left(\Omega_{f}^{-1}\right)$ time scale, the same qualitative features are recovered, but typically the eruption of the boundary layer is delayed to rather later times. However, a different small-time behaviour in the rotation rate of the container would modify somewhat the asymptotic scalings associated with the onset of a centrifugal instability, as described by (6.7), primarily because the base flow would no longer be an impulsive Rayleigh layer. 
We conclude by reiterating that this system provides an ideal test-bed for a detailed investigation of the role of boundary-layer singularities, and associated instabilities in fluid mechanics. The closed nature of the flow domain means that the boundary conditions relevant to computation of the Navier-Stokes system are all well defined, with no modelling of inflow/outflow conditions required. Similarly the structure of the boundary layer lends itself to asymptotic description in the axisymmetric regime. In this sense it is an ideal problem for a combined attack by numerical, asymptotic and experimental methods.

The authors are grateful to Professor T. Mullin for suggesting this problem and for many useful discussions. The authors would also like to thank P. Hurley for his contribution of the linearised Navier-Stokes elements to oomph-lib. Parts of this work were completed whilst R.E.H. was visiting the University of Auckland and the University of Adelaide, and he gratefully acknowledges their support. R.J.C. is supported by the Marsden Fund Council, administered by the Royal Society of New Zealand.

\section{Appendix. The governing equations}

In the coordinate system of figure 1, the dimensionless Navier-Stokes reduce to:

$$
\begin{aligned}
& \frac{\partial u}{\partial t}+u \frac{\partial u}{\partial r}+\frac{v}{r} \frac{\partial u}{\partial \theta}+\frac{\delta w}{h} \frac{\partial u}{\partial \phi}-\frac{v^{2}}{r}-\frac{\delta \cos \theta}{h} w^{2}=-\frac{\partial p}{\partial r} \\
& -E k\left\{\frac{1}{r h} \frac{\partial}{\partial \theta}\left[h\left(\frac{\partial v}{\partial r}+\frac{v}{r}-\frac{1}{r} \frac{\partial u}{\partial \theta}\right)\right]-\frac{\delta^{2}}{h^{2}} \frac{\partial^{2} u}{\partial \phi^{2}}+\frac{\delta}{h}\left(\frac{\partial}{\partial \phi} \frac{\partial w}{\partial r}+\frac{\delta \cos \theta}{h} \frac{\partial w}{\partial \phi}\right)\right\}, \\
& \frac{\partial v}{\partial t}+u \frac{\partial v}{\partial r}+\frac{v}{r} \frac{\partial v}{\partial \theta}+\frac{\delta w}{h} \frac{\partial v}{\partial \phi}+\frac{u v}{r}+\frac{\delta \sin \theta}{h} w^{2}=-\frac{1}{r} \frac{\partial p}{\partial \theta} \\
& +E k\left\{\frac{1}{h} \frac{\partial}{\partial r}\left[h\left(\frac{\partial v}{\partial r}+\frac{v}{r}-\frac{1}{r} \frac{\partial u}{\partial \theta}\right)\right]+\frac{\delta^{2}}{h^{2}} \frac{\partial^{2} v}{\partial \phi^{2}}-\frac{\delta}{h r} \frac{\partial}{\partial \theta} \frac{\partial w}{\partial \phi}+\frac{\delta^{2} \sin \theta}{h^{2}} \frac{\partial w}{\partial \phi}\right\}, \\
& \frac{\partial w}{\partial t}+u \frac{\partial w}{\partial r}+\frac{v}{r} \frac{\partial w}{\partial \theta}+\frac{\delta w}{h} \frac{\partial w}{\partial \phi}+\frac{\delta \cos \theta}{h} u w-\frac{\delta \sin \theta}{h} v w=-\frac{\delta}{h} \frac{\partial p}{\partial \phi} \\
& +E k\left\{\frac{1}{r^{2}} \frac{\partial}{\partial \theta}\left[\frac{1}{h}\left(h \frac{\partial w}{\partial \theta}-\delta r \sin \theta w\right)\right]+\frac{1}{r} \frac{\partial}{\partial r}\left[\frac{r}{h}\left(h \frac{\partial w}{\partial r}+\delta \cos \theta w\right)\right]\right. \\
& \left.-\left(\frac{\partial}{\partial r}+\frac{1}{r}\right) \frac{\delta}{h} \frac{\partial u}{\partial \phi}-\frac{1}{r} \frac{\partial}{\partial \theta}\left(\frac{\delta}{h} \frac{\partial v}{\partial \phi}\right)\right\}, \\
& \frac{\partial u}{\partial r}+\frac{(1+2 \delta r \cos \theta)}{r h} u+\frac{1}{r} \frac{\partial v}{\partial \theta}-\frac{\delta \sin \theta}{h} v+\frac{\delta}{h} \frac{\partial w}{\partial \phi}=0 .
\end{aligned}
$$

In this formulation, $h=(1+\delta r \cos \theta)$ is a dimensionless measure of the distance to the central axis of rotation, where $r=r^{*} / a$, and $\delta=a / L$ is a curvature parameter. The Ekman number is defined by $E k=v /(U a)$, where $v$ is the kinematic viscosity of the fluid and $U=a \Omega_{f}$ is a relevant velocity scale. In the cross-sectional plane, the velocity components are $(u, v, w)$ in the coordinate system $(r, \theta, \phi)$. 


\section{REFERENCES}

BANKs, W. H. H. \& ZATURSKA, M. B. 1979 The collision of unsteady laminar boundary layers. J. Engng Maths 13 (3), 193-212.

Benton, E. R. \& Clark, A. 1974 Spin-up. Annu. Rev. Fluid Mech. 6, 257-280.

Berger, S. A., Talbot, L. \& YaO, L. S. 1983 Flow in curved pipes. Annu. Rev. Fluid Mech. 15 (1), 461-512.

Cowley, S. J., Van Dommelen, L. L. \& Lam, S. T. 1990 On the use of Lagrangian variables in descriptions of unsteady boundary-layer separation. Phil. Trans. R. Soc. Lond. 333 (1631), 343-378.

Demkowicz, L., Oden, J. T., Rachowicz, W. \& Hardy, O. 1989 Toward a universal h-p adaptive finite element strategy, Part 1. Constrained approximation and data structure. Comput. Meth. Appl. Mech. Engng 77, 79-112.

Denier, J. P., Hall, P. \& Seddougui, S. O. 1991 On the receptivity problem for Görtler vortices: vortex motions induced by wall roughness. Phil. Trans. R. Soc. Lond. 335 (1636), $51-85$.

Elman, H. C., Silvester, D. J. \& Wathen, A. J. 2005 Finite Elements and Fast Iterative Solvers: With Applications in Incompressible Fluid Dynamics. Oxford University Press.

HALl, P. 1985 The Görtler vortex instability mechanism in three-dimensional boundary layers. Proc. R. Soc. Lond. A 399 (1816), 135-152.

HALL, P. 1990 Görtler vortices in growing boundary layers: the leading edge receptivity problem, linear growth and the nonlinear breakdown stage. Mathematika 37, 151-189.

HeIL, M. \& HAZEL, A. L. 2006 oomph-lib - an object-oriented multi-physics finite-element library. In Fluid-Structure Interaction (ed. M. Schafer \& H.-J. Bungartz). Lecture Notes on Computational Science and Engineering, pp. 19-49. Springer.

Heroux, M. A., Bartlett, R. A., Howle, V. E., Hoekstra, R. J., Hu, J. J., Kolda, T. G., Lehoucq, R. B., Long, K. R., Pawlowski, R. P., Phipps, E. T., SAlinger, A. G., Thornquist, H. K., Tuminaro, R. S., Willenbring, J. M., Williams, A. \& StAnley, K. S. 2005 An overview of the trilinos project. ACM Trans. Math. Softw. 31 (3), 397-423.

Mackerrell, O., Blennerhassett, P. J. \& Bassom, A 2002 Görtler vortices in the Rayleigh layer on an impulsively started cylinder. Phys. Fluids 14 (9), 2948-2956.

Madden, F. N. \& Mullin, T. 1994 The spin-up from rest of a fluid-filled torus. J. Fluid Mech. 265, 217-244.

Oтто, S. R. 1993 Stability of the flow around a cylinder: the spin-up problem. IMA J. Appl. Maths 51 (1), 13-26.

Del Pino, C., Hewitt, R. E., Clarke, R. J., Mullin, T. \& Denier, J. P. 2008 Unsteady fronts in the spin-down of a fluid-filled torus. Phys. Fluids 20 (12), 124104.

Siggers, J. H. \& Waters, S. L. 2005 Steady flows in pipes with finite curvature. Phys. Fluids 17, 077102.

Siggers, J. H. \& WATERS, S. L. 2008 Unsteady flows in pipes with finite curvature. J. Fluid Mech. 600, 133-165.

Simpson, C. J. \& Stewartson, K. 1982 A note on a boundary-layer collision on a rotating sphere. Z. Angew. Math. Phys. 33 (3), 370-378.

Stewartson, K., Cebeci, T. \& Chang, K. C. 1980 A boundary-layer collision in a curved duct. Q. J. Mech. Appl. Maths 33 (1), 59-75.

YANG, Z.-H. \& Keller, H. 1986 Multiple laminar flows through curved pipes. Appl. Numer. Maths 2, 257-271.

ZIENKIEWICZ, O. C \& ZHU, J. Z $1992 a$ The superconvergent patch recovery and a posteriori error estimates. Part 1: The recovery technique. Intl J. Numer. Meth. Engng 33 (7), 1331-1364.

ZIENKIEWICZ, OC \& ZHU, JZ $1992 b$ The superconvergent patch recovery and a posteriori error estimates. Part 2: Error estimates and adaptivity. Intl J. Numer. Meth. Engng 33 (7), $1365-1382$.

ZuRigat, Y. H. \& MALIK, M. R. 1995 Effect of cross-flow on Görtler instability in incompressible boundary layers. Phys. Fluids 7, 1616. 